\title{
Stochastic reduced-order models for stable nonlinear ordinary differential equations
}

\author{
Alin Radu $(\mathbb{D}$
}

Received: 12 April 2018 / Accepted: 19 April 2019 / Published online: 7 May 2019

(C) The Author(s) 2019

\begin{abstract}
Two methods based on stochastic reducedorder models (SROM) are proposed to solve stochastic stable nonlinear ordinary differential equations. One general method available for the probabilistic characterization of the response of nonlinear systems subjected to random excitation is Monte Carlo (MC), wherein the response of the nonlinear system must be calculated for a large number of samples of the input, which can be very computationally demanding. Random vibration theory is also inadequate for calculating response statistics for both linear systems under non-Gaussian inputs and nonlinear systems subjected to any kind of excitation. The two methods proposed are based on SROM, i.e., stochastic models with a finite number of optimally selected samples. The first method uses a SROM model for the random input. The second method is based on a surrogate model for the response of the nonlinear system defined on a Voronoi tessellation of the input samples. The newly proposed methods are applied for stable nonlinear ordinary differential equations, with deterministic coefficients and stochastic input, that are used in engineering applications: single-degree-of-freedom Duffing and BoucWen systems, and a two-degree-of-freedom nonlinear energy sink system. The numerical results suggest that SROMs are able to estimate statistics of the stochastic responses for these systems efficiently and accurately, results validated by the benchmark MC results.
\end{abstract}

A. Radu $(\varangle)$

University of Bristol, Bristol BS8 1TR, UK

e-mail: alin.radu@bristol.ac.uk
Keywords Stochastic reduced-order models . Stochastic nonlinear dynamic equations - Stochastic processes $\cdot$ Response statistics $\cdot$ Extreme values

\section{Introduction}

Random vibration theory and numerical methods to date provide efficient solutions for second-moment properties of the states of arbitrary linear systems subjected to Gaussian random input. Unless the input is Gaussian and the system is linear or the output can be assumed to be Gaussian, the first two moments of the response are insufficient for calculating response statistics [1] (Sect. 5.3). This is a significant limitation since realistic dynamic systems behave nonlinearly under strong random vibrations and the distribution of the response is non-Gaussian. For example, the distribution of the pressure field acting on a spacecraft during atmospheric re-entry is highly nonGaussian [2]; the assumption that the responses of structures under extreme wind [3] or seismic [4] excitations are linear may be inadequate; the aircraft main landing-gears are nonlinear complex systems, which require time-consuming dynamic simulations to calculate steady-state solutions [5]. Thus, the reliability of such structures cannot be obtained within the framework of the random vibration theory. The conceptually simple framework of the random vibration theory cannot be used in such complex applications since some concepts cannot even be defined. The first- and 
second-moment equations for linear systems in random vibration theory are formal since it views white noise as a process with constant spectral density, i.e. a process with infinite variance, which is not defined in the second-moment sense [6] (Sect. 7.2).

Monte Carlo (MC) is one general and reliable method available for solving complex nonlinear dynamic systems subjected to complex stochastic input, i.e. non-Gaussian, non-stationary non-white noise. The method is asymptotically correct since it approaches the exact solution as the number of simulations increases indefinitely. However, $\mathrm{MC}$ simulation is computationally impractical for complex realistic nonlinear systems $[5,7,8]$ since it involves repeated deterministic dynamic analyses for randomly selected samples of the random excitation. Efficient alternatives to the MC method have been studied before, such as the quasi-Monte Carlo simulation [7,9-11], Latin hypercube $[12,13]$, or change of measure [6] (Sect. 5.4). The limitation of the MC method has encouraged the development of approximate methods for finding statistical properties of the dynamic systems under random excitation. Some approaches are based on heuristic arguments, such as the equivalent stochastic linearisation [14-16], while others rely on rigorous but rather restrictive conditions, as in the stochastic averaging method [17-19] or perturbation [1] (Sect. 6.2), [20]. Other well-tested methodologies used for the calculation of response statistics of nonlinear systems involve the use of the Fokker Plank equation [21-23], moments and cumulant closures [24,25], stochastic modelling using polynomial chaos [26,27], or reduced orders of the nonlinear system by using linear or nonlinear normal modes [28-30]. This enumeration of methods used for solving stochastic nonlinear equations is not exhaustive, but so far using these approaches proved to make capturing statistics of extreme responses of nonlinear systems difficult or even impossible due to their built-in limitations [31], because they have solutions only for special cases [32], or are too computationally expensive [33].

The goal of this paper is to propose novel methods that can be used to calculate response statistics of engineering systems, characterised by stable, nonlinear ordinary differential equations with deterministic coefficients and general non-Gaussian, non-stationary stochastic input. Even though the methodology proposed is general for the described setup, the study of complex systems such as turbulent or chaotic systems, or infinite-dimensional systems with multiple instabilities is beyond the scope of this paper. However, considerable advances on developing efficient methods to quantify the uncertainty in complex stochastic highly nonlinear systems like Earth's climate or oceans $[28,34]$ must be acknowledged. The two methods proposed in the current study to solve the aforementioned engineering systems are conceptually simple, accurate, non-intrusive and computationally efficient and are based on stochastic reduced-order models (SROMs) [35]. Stochastic reduced-order models are stochastic processes that have a finite number of samples selected in an optimal manner from the samples of the target process. Like MC simulation, the methods use samples of seismic load processes to characterize structural response and are not intrusive in the sense that their construction uses deterministic solutions. However, the proposed SROMs use small numbers of input samples selected in an optimal manner. In contrast, MC simulation uses a large number of samples selected at random. The use of optimally selected samples allows to reduce the number of simulations required by the MC method by one or two orders of magnitude while retaining accuracy. The method has been originally developed for dynamic response [36]. It has been shown that for static problems the SROM-based method can be improved significantly [37], and an adaptive method to further refine SROMs used in solving stochastic equations was proposed in [38]. Recently, SROMs have already been used successfully in applications for the quantification of the uncertainty in electromagnetic-signals interference in cables [39], or in the inter-granular corrosion rates [40]. SROMs have also been used in solving inverse problems, with applications in the identification of material properties in elastodynamics [41], traditionally solved using Bayesian inferences or stochastic-optimisation approaches.

The first method presented in this paper, referred to as the SROM method, constructs a SROM using directly the input samples. The second method, referred to as the extended SROM method, i.e., the ESROM method, builds upon the SROM method to constructs an SROM-based surrogate model for the response of the dynamic system, defined on a Voronoi tessellation of the input samples. Both methods proposed in this paper may be used as alternatives to $\mathrm{MC}$ for calculating response statistics for dynamic nonlinear equations, with just a fraction of the computational effort 
required by MC. The SROM method provides quick response statistics within some error of the MC results, while the superior ESROM method can provide reliable results even in the extreme tails of the response probability distributions. Thus, the ESROM would allow for a simulation-based design of highly reliable nonlinear engineering systems for probability of no-collapse of the $10^{-6}$ order for earthquake-resistant structures [42], or probability per flight hour of catastrophic failure for aircrafts as low as $10^{-9}$ [43], probabilities that otherwise would require billions or even trillions of MC dynamic analyses [7]. Applications of the methods proposed are shown for simple second-order, oneand two-degree-of-freedom nonlinear dynamic ordinary differential equations (ODEs) with deterministic coefficients and stochastic input. Response statistics such as probability tail distributions and stochastic moments are compared with the reference MC results.

\section{Nonlinear dynamic system definition}

Nonlinear dynamic systems subjected to random input can be represented by nonlinear stochastic ODEs with the general form $\sum_{j=0}^{N} \Psi_{j}\left(t, X(t), Y^{(j)}(t)\right)=0, t \geq$ 0 , where $\Psi_{j}$ are linear or nonlinear differentiable functions of time $t$ with deterministic or stochastic coefficients; $Y^{(j)}(t)=d^{j} Y(t) / d t^{j}, j=1, \ldots, N$ are the $j$ th derivatives with respect to time of the state $Y(t)$ of the dynamic system; and $X(t)$ is the stochastic input. Functions $\Psi_{j}$ for the example systems in Eqs. (2-5) used to demonstrate how the proposed framework works, have deterministic coefficients, and are power, or exponential functions, but any other nonlinear differential function would work too. The response of the nonlinear dynamic system is described by the state $Y(t)$, for given initial conditions $Y^{(k)}(0), k=1, \ldots, n-1$.

The stochastic process $X(t)$ can be any stochastic process, and thus we assume a type of process that fits the general case of a zero-mean, non-Gaussian, nonstationary process:

$X(t)=f(t)\left[F_{T}^{-1} \circ \Phi(G(t ; g(v)))\right]$,

where $g(v)=\left(\nu \theta_{2} \sqrt{2 \pi}\right)^{-1} \exp \left\{-0.5\left(\ln (\nu)-\theta_{1}\right)^{2} / \theta_{2}^{2}\right\}$ is a one-sided power spectral density function, with parameters $\left(\theta_{1}, \theta_{2}\right)$, that provides the second-order moment properties and the frequency content of $X(t)$; $f(t)=\theta_{3} t^{\theta_{4}} \exp \left(\theta_{5} t\right)$ is an amplitude-modulation function with parameters $\left(\theta_{3}, \theta_{4}, \theta_{5}\right)$ that describes the non-stationary character of $X(t) ; \Phi($.$) is the cumulative$ probability distribution function for the standard normal distribution; $G(t ; g(v))$ is a standard Gaussian process with second-order moment properties given by the function $g(v)$; and $F_{T}\left(. ; n_{T}\right)$ is the Student's T distribution with $n_{T}$ degrees of freedom. Functions $f(t), g(v)$ and $F_{T}\left(. ; n_{T}\right)$ are customary and define the probability law of $X(t)$, but can be replaced by any other functions that describe the amplitude non-stationarity character, the frequency content, and the distribution of $X(t)$, respectively. Function $f(t)$ is called a Gamma function and has been used before to describe, for example, the non-stationarity in seismic ground motions [44]. The choice of the Student's T distribution for $X(t)$ is justified by its heavy tails specific to heavy-tailed phenomena to which engineering systems are subjected, such as: offshore structures subjected rough waves [45], high-rise structures subjected to strong wind [46], or earthquake [4] loads. The distribution $F_{T}\left(. ; n_{T}\right)$ can be replaced by other more complex, multi-modal distributions. However, for the purpose of loads with high peaks, the Student's T distribution has a kurtosis higher than 3 that can be fit to data, and thus experience tails higher than Gaussian processes, similar to realistic loads with this characteristics, such as: the wind pressure on low-rise structures tested in wind tunnels [47]; the coastal-wave elevations measured in Duck, North Carolina [48]; the seismic ground-acceleration for earthquakes recorded on rock sites [44]; or the unevenness of a railway track in India [49]. The representation of non-Gaussian processes in Eq. (1) is known as a memoryless transformation [50] and provides an intuitive way to simulate samples of non-Gaussian processes. Numerical examples are shown for the scalar parameters $\left(\theta_{1}, \ldots, 5\right)=(2,0.5,0.5,1.5,-0.35)$ and $n_{T}=3$. Note that the process $X(t)$ has variance $\int_{\nu \geq 0} g(v) d v=1$, which can also be customised to a value $\sigma_{X}^{2}$, by multiplying $g(v)$ by it. Figure $1 \mathrm{a}, \mathrm{b}$ shows the function $g(v)$ for two sets of parameters $\left(\theta_{1}, \theta_{2}\right)=\{(2,0.5),(1,1)\}$, and corresponding samples of $X(t)$, respectively.

Three simple nonlinear ODEs with various applications in engineering are used as examples. The first system is the single-degree-of-freedom (SDOF) Duffing oscillator $[51,52]$ used, for example, in modelling 
Fig. 1 a Power spectral density function $g(v)$, b samples of the input process $X(t)$ (a)

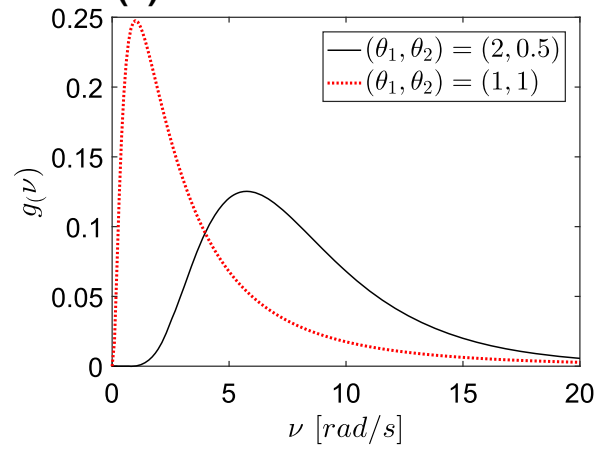

(b)

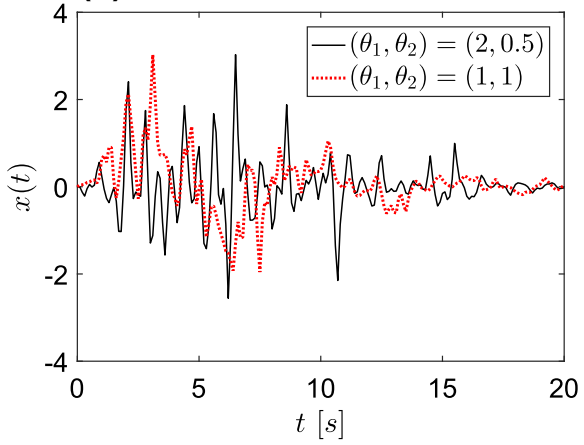

vibration-control systems with cubic stiffness [53] in mechanical engineering:

$\ddot{Y}(t)+2 \zeta \nu \dot{Y}(t)+v^{2}\left(Y(t)+\rho Y^{3}(t)\right)+X(t)=0$.

The second system described by the following ODEs is the Bouc-Wen SDOF system [54,55] used, for example, to model buildings' seismic ductility demand [56] in civil engineering:

$$
\begin{aligned}
& \ddot{Y}(t)+2 \zeta v \dot{Y}(t)+v^{2}(\rho Y(t) \\
& \quad+(1-\rho) W(t))+X(t)=0 \\
& \dot{W}(t)=-\gamma \dot{Y}(t)+\alpha|W(t)|^{\eta-1} W(t)+\beta \dot{Y}(t)|W(t)|^{\eta},
\end{aligned}
$$

where the process $W(t)$ is known as the hysteresis response of the Bouc-Wen oscillator. Finally, the last system solved as an example for the proposed methodologies is the two-degree-of-freedom (2DOF) nonlinear energy sink (NES) system [57], used for the reduction of the response of ocean-engineering systems subjected to extreme loading [58], whose state is described by the bi-variate stochastic process $Y(t)=$ $\left[Y_{1}(t), Y_{2}(t)\right]$ defined by:

$$
\begin{aligned}
& \ddot{Y}_{1}(t)+2 \zeta v \dot{Y}_{1}(t)+v^{2} Y_{1}(t)+\lambda_{1}\left(\dot{Y}_{1}(t)-\dot{Y}_{2}(t)\right) \\
& \quad+c_{1}\left(Y_{1}(t)-Y_{2}(t)\right)^{3}+X(t)=0 \\
& \ddot{Y}_{2}(t)+\lambda_{2}\left(\dot{Y}_{2}(t)-\dot{Y}_{1}(t)\right) \\
& \quad+c_{2}\left(Y_{2}(t)-Y_{1}(t)\right)^{3}+X(t)=0 .
\end{aligned}
$$

In all three systems of ODEs in Eqs. (2-5), $\dot{Y}(t)=$ $d Y(t) / d t$ and $\ddot{Y}(t)=d^{2} Y(t) / d t^{2}$ are the first- and second-order derivatives of the systems' states $Y(t)$ with respect to time $t$, and zero initial conditions are assumed. The following systems' parameters $v=\pi$, $\zeta=0.1, \alpha=4, \beta=-7, \gamma=0.25, \rho=0.3, \eta=2$, $c_{1}=1, c_{2}=9, l_{1}=0.1$ and $l_{2}=0.9$ were used for the numerical examples shown in the remainder of the paper. The goal of the paper is to calculate statistics of the responses $Y(t)$ of nonlinear equations using two novel and efficient computational methods based on stochastic reduced-order models (SROMs), that could substitute reliably the solutions provided by the traditional MC simulations. The ODEs in Eqs. (2-5) are used just as examples to illustrate the benefits and limitations of the methodologies proposed.

\section{Solutions and response statistics}

Monte Carlo (MC) simulation is a general method available for finding statistical properties of the state $Y(t)$ of an arbitrary nonlinear dynamic system subjected to stochastic input. However, the method is impractical for complex systems due to its high computational cost. The objective of this paper is to adopt practical, accurate and efficient methods for calculating statistics of the response $Y(t)$ of nonlinear dynamic systems under general stochastic excitation. We propose two novel methods for calculating response statistics of the response $Y(t)$ of nonlinear dynamic systems subjected to stochastic input $X(t)$. Both methods are based on stochastic reduced-order models (SROMs) for the input: one, more straightforward, uses the SROM directly to find the response of the system for a small number of optimally selected samples; the second one, referred to as the extended SROM, uses the input SROM to construct a surrogate model for the system's response based on a first-order Taylor expansion. A SROM of $X(t)$ is a stochastic process $\tilde{X}(t)$ represented 
by a finite number $m$ of samples $\tilde{x}_{k}(t)$ of $X(t)$ with probabilities $p_{k}, k=1, \ldots, m$, such that the probability laws of $X(t)$ and $\tilde{X}(t)$ are similar in some sense.

The SROM-based methods will be tested against MC results, through the comparison of statistics of the state $Y(t)$. In this case, we will aim to compare the tail distributions and high-order moments. MC method requires the calculation of the response $y_{k}(t)$ for a large number $n$ of input samples $x_{k}(t), k=1, \ldots, n$. The moments of order $q$, and the tail distribution function can be estimated using the MC response samples, respectively, as:

$$
\begin{aligned}
\mathbf{E}\left[Y(t)^{q}\right] & =\frac{1}{n} \sum_{k=1}^{n} \tilde{y}_{k}(t)^{q}, \\
F(y) & =\mathbf{P}(Y(t)>y) \\
& =\frac{1}{n} \sum_{k=1}^{n} \mathbf{1}\left(\max _{t \geq 0}\left|\tilde{y}_{k}(t)\right|>y\right),
\end{aligned}
$$

where 1(.) is the indicator function. The two methods proposed are presented in the following two sections. The first one, involves the construction of a SROM $\tilde{X}(t)$ for the input $X(t)$, with $m<<n$ samples. The second method, referred to as the extended SROM (ESROM), constructs a surrogate model $\tilde{Y}(t)$ for the state $Y(t)$ of the system, based on $\tilde{X}(t)$.

\subsection{SROM-based solution}

Let $\left\{\left(x_{l}(t), l=1, \ldots, n\right\}\right.$ be a large enough set of samples of the input process $X(t)$ that can characterise its probability law. Any number $m<<n$ of samples $\left\{\left(\tilde{x}_{k}(t), p_{k}\right), k=1, \ldots, m\right\}$, selected from $\left\{\left(x_{l}(t), l=1, \ldots, n\right\}\right.$, with probabilities $p_{k}$ such that $\sum_{k=1}^{m} p_{k}=1$ define a stochastic reduced-order model (SROM) $\tilde{X}(t)$ of $X(t)$. Similar to MC, the SROM also uses random samples of $X(t)$, but unlike in the case of MC they are not equally likely, but weighed by distinct probabilities. It is shown in $[36,59]$ that it is possible to select a small number $m$ of samples $\left\{\left(\tilde{x}_{k}(t), p_{k}\right), k=1, \ldots, m\right\}$ of $X(t)$ such that $X(t)$ and $\tilde{X}(t)$ have similar probability laws. We are looking for a $\operatorname{SROM} \tilde{X}(t)$ with a range $\left\{\tilde{x_{k}}(t), k=1, \ldots, m\right\}$ of a relatively small number $m$ of independent samples of $X(t)$. Our objective is to find $p_{k}$ such that the discrepancies between the probability laws of the SROM
$\tilde{X}(t)=\left\{\left(\tilde{x}_{k}(t), p_{k}\right), k=1, \ldots, m\right\}$ and $X(t)$ are minimized [60]. Processes $\tilde{X}(t)$ and $X(t)$ are defined on the same probability space, and in order for the SROM $\tilde{X}(t)$ to characterize the original model $X(t)$, the $m$ samples selected need to be largely spaced from each other in order to explore the entire range of $X(t)$ samples, rather than being clustered in small subsets. Algorithms such as the integer optimisation [61], dependent thinning [62] or pattern classification [63] may be used to select the samples that define $\tilde{X}(t)$. The probability laws of stochastic processes are defined by their moments, marginal distributions and covariance functions. Target moments $\mu_{X}(t ; q)$ of order $q$, marginal distribution $F_{X}(x ; t)$ and covariance function $\Sigma_{X}(t, s)$ of $X(t)$ can be calculated directly from its probability law, if available, or from its samples. The statistics for the SROM $\tilde{X}(t)$ are estimated from a selection of $m$ samples, weighed by their probabilities. Thus, the moments $\tilde{\mu}_{X}(t ; q)$ of order $q$, the marginal distribution $\tilde{F}_{X}(x ; t)$ and the covariance function $\tilde{\Sigma}_{X}(t, s)$ of the $\operatorname{SROM} \tilde{X}(t)$ are calculated, respectively, as:

$$
\begin{aligned}
& \tilde{\mu}_{X}(t ; q)=\sum_{k=1}^{m} p_{k} \tilde{x}_{k}^{q}(t), \\
& \tilde{F}_{X}(x ; t)=\sum_{k=1}^{m} p_{k} \mathbf{1}\left\{\tilde{x}_{k}(t) \leq x\right\}, \\
& \tilde{\Sigma}_{X}(t, s)=\sum_{k=1}^{m} p_{k} \tilde{x}_{k}(t) \tilde{x}_{k}(s) .
\end{aligned}
$$

Note that $p_{k}$ are the likelihood probabilities assigned to the input samples $\tilde{x}_{k}(t)$ of the SROM and they have the following properties: (1) if $m$ is very large and $\tilde{x}_{k}$ are independent samples of $X$, then optimal probabilities $p_{k} \approx 1 / m$, as it approaches the case of Monte Carlo when all samples are equally likely; (2) the moments $\tilde{\mu}_{X}(t ; q)$ and distribution $\tilde{F}_{X}(x ; t)$ of $\tilde{X}(t)$ are unbiased estimators of the corresponding statistics of $X(t)$ with variances depending on the range of $\tilde{X}(t)$; and (3) if $Y(t)=h(X(t))$ is the solution of a stochastic algebraic, differential or integral equation depending on $X(t)$, the solution $\tilde{Y}(t)$ of this equation with $\tilde{X}(t)$ in place of $X(t)$ is given by the samples $\tilde{y}_{k}(t)=h\left(\tilde{x}_{k}(t)\right)$ with probabilities $p_{k}$. The last property is essential for the success of the SROM method, and its proof relies on the argument that if the probabilities $p_{k}$ partition the probability space of the input $X(t)$, then the expected solution $h(X(t))$ in a given partition char- 
acterised by $\left(\tilde{x}_{k}(t), p_{k}\right)$ is given by the deterministic solution $\tilde{y}_{k}(t)=h\left(\tilde{x}_{k}(t)\right)$ with probability $p_{k}$. More comprehensive arguments and detailed proves using measure theory for these properties are presented in [35]. Optimization algorithms for the construction of SROMs have been developed in $[35,41]$. A least mean square algorithm is used to identify the optimal probabilities $p_{k}$ that minimise the discrepancies between $X(t)$ and $\tilde{X}(t)$ herein, for a given cardinal $m$ and a set of samples $\tilde{x}_{k}(t)$. Based on the aforementioned property (1), the initial values of $p_{k}=1 / m$ are assumed as the starting point in the optimisation algorithm.

Figure 2 shows the first two-order moments $\tilde{\mu}_{X}(t ; q), q=1,2$ of the SROM $\tilde{X}(t)$ for (a) $m=20$ and (b) $m=10^{3}$, in comparison with the respective MC moments $\mu_{X}(t ; q), q=1,2$ calculated from $n=10^{4}$ samples. In order to already see the advantaged of SROMs, Fig. 2c, d shows 30 trials of calculating the same moments $\mu_{X}(t ; q), q=1,2$ using MC, but just with a limited number of independent equally likely samples $n=20$ and $n=10^{3}$, respectively. By comparing panels (a) with (c), and (b) with (d), respectively, we can see that unlike SROM, MC is incapable of capturing the properties of $X(t)$ with just a few samples, the variability between the estimated of the moments being significant. Similar observations about the efficiency of the SROM vs. MC with the same small number of samples can be done regarding to the marginal distributions and the covariance functions, but due to the space limitations and given the scope of the paper, such additional preliminary results are not presented. Figure 3 shows (a) the marginal distribution of $X(t)$ calculated by MC with $n=10^{4}$ samples, and the marginal distributions $\tilde{F}_{X}(x, t)$ of the SROM $\tilde{X}(t)$ for (b) $m=20$ and (c) $m=10^{3}$. Similarly, Fig. 4 shows (a) the covariance of $X(t)$ calculated by MC with $n=10^{4}$ samples, and the covariance functions $\tilde{\Sigma}_{X}(t, s)$ of the $\operatorname{SROM} \tilde{X}(t)$ for (b) $m=20$ and (c) $m=10^{3}$.

Finally, following the property (3) of the SROM samples $\left(\tilde{x}_{k}(t), p_{k}\right)$, the moments of order $q$ and the tail distribution of the system's response $Y(t)$ shown in Eqs. (6) and (7) can be computed by the following proxies using the samples $\tilde{y}_{k}(t), k=1, \ldots, m$ calculated as the response of Eqs. (2-5) to the samples $\tilde{x}_{k}(t)$ of the SROM $\tilde{X}(t)$ :

$\mathbf{E}\left[Y(t)^{q}\right]=\sum_{k=1}^{m} p_{k} \tilde{y}_{k}(t)^{q}$,

$$
\begin{aligned}
F(y) & =\mathbf{P}(Y(t)>y) \\
& =\sum_{k=1}^{m} p_{k} \mathbf{1}\left(\max _{t \geq 0}\left|\tilde{y}_{k}(t)\right|>y\right) .
\end{aligned}
$$

Note that only $m$ deterministic dynamic analyses are needed to obtain the solution of $Y(t)$ in this case, i.e. the solution for Eqs. (2-5) to the $m$ samples of the SROM $\tilde{X}(t)$. The essential benefit of the method consists in the fact that the SROM $\tilde{X}(t)$ is built for the input $X(t)$ and that the only dynamic analyses needed are the one for the samples $\tilde{x}_{k}$ with probabilities $p_{k}$ since they characterise the probability law of $X(t)$. It must also be emphasized that the pairs $\left(\tilde{x}_{k}(t), p_{k}\right)$ describe fully the probability law of the SROM $\tilde{X}(t)$.

\subsection{ESROM-based solution}

The ESROM method develops a surrogate model $\tilde{Y}(t)$ for the state $Y(t)$ of the nonlinear ODEs employing the following four steps. First, a parametric mode for the input process $X(t)$ is constructed:

$X(t) \simeq \sum_{i=1}^{d} Z_{i} \varphi_{i}(t)$,

where $Z=\left\{Z_{i}, i=1, \ldots, d\right\}$ is a random vector and $\left\{\varphi_{i}(t),, i=1, \ldots, d\right\}$ are deterministic specified functions of time. Parametric models of this type can be calculated for any stochastic process. We use a truncated Karhunen-Loève model to calculate functions $\left\{\varphi_{i}(t), i=1, \ldots, d\right\}$ by calibrating it to the first two moments of $X(t)$, that is, the mean and covariance functions [64]. Functions $\varphi_{i}(t)$ are the eigenvectors of the covariance matrix $\Sigma(t, s)$ defined for $0 \leq t, s \leq \tau$. Unlike the Karhunen-Loève expansion, for which $X(t)=\lim _{d \rightarrow \infty} \sum_{i=1}^{d} \sqrt{\lambda_{i}} Z_{i} \varphi_{i}(t), t \geq 0$, where $\lambda_{i}$ are the eigenvectors and $Z_{i}$ are random variables with zero mean and unit variance, the random vector $Z$ is characterized by its samples. Samples $z_{k, i}$ of $Z_{i}$ are obtained by minimizing the difference between the actual samples $x_{k}(t)$ of $X(t)$ and their approximations $\sum_{i=1}^{d} z_{k, i} \varphi_{i}(t)$, where $z_{k, i}$ denotes the $i$-th component of sample $z_{k}$ of $Z$. The parametric model $\sum_{i=1}^{d} Z_{i} \varphi_{i}(t)$ is converging to the original process $X(t)$ as the dimension $d$ of the vector $Z$ increases. Figure 5 shows one sample of $X(t)$ and its approximation for two values (a) $d=50$, and (b) $d=100$. 
In the second step, we construct a SROM $\tilde{Z}=$ $\left\{\left(\tilde{z}_{k}, p_{k}\right), k=1, \ldots, m\right\}$ for $Z$ using the samples of the random vector $Z$, following the procedure presented in the SROM-based solution, in Sect. 3.1. Note that $n$ samples of $z_{k}$ of $Z$ are available, one for each sample of $x_{k}(t)$ of $X(t)$. As discussed previously, we seek to find optimum probabilities $p_{k}, k=1, \ldots, m$ for each sample $\tilde{z}_{k}$ defining the SROM $\tilde{Z}$ of $Z$, with $\sum_{k=1}^{m} p_{k}=1$, such that the probability laws of the $d$-dimensional random vectors $Z$ and $\tilde{Z}$ are similar.

In the third step, we construct a Voronoi tessellation $\left\{\Gamma_{k}, k=1, \ldots, m\right\}$ on the range of $Z$, with centres $\left\{\tilde{z}_{k}\right\}[37,38]$. Each cell $\Gamma_{k}$ contains all samples $z_{j}$ of $Z$ that are closest to its centre, i.e. $\Gamma_{k}=\left\{z_{j}:\left|z_{j}-\tilde{z}_{k}\right| \leq\right.$ $\left.\left|z_{j}-\tilde{z}_{l}\right|, l \neq k\right\}$. Note that the probability $p_{k}$ defines also the probability that $Z$ takes values in cell $\Gamma_{k}$.

Finally, in the fourth step, a surrogate model $\tilde{Y}(t ; Z)$ for $Y(t)$ is constructed using local piece-wise linear approximations of $Y(t ; Z)$ for each Voronoi cell by using a first-order Taylor expansion at the centre $Z=$ $z_{k}$ of the cell:

$$
\begin{aligned}
& \tilde{Y}(t ; Z) \\
& \quad=\sum_{k=1}^{m} \mathbf{1}\left\{Z \in \Gamma_{k}\right\}\left[Y\left(t ; \tilde{z}_{k}\right)+\nabla Y\left(t ; \tilde{z}_{k}\right)\left(Z-\tilde{z}_{k}\right)\right],
\end{aligned}
$$

where $Y\left(t ; \tilde{z}_{k}\right)$ is the response $Y(t)$ corresponding to the pair $\left(\tilde{z}_{k}, p_{k}\right)$ and $\nabla Y\left(t ; \tilde{z}_{k}\right)=\left\{\partial Y(t ; Z) / \partial Z_{i} \mid Z=\right.$ $\left.\tilde{z}_{k}, i=1, \ldots, d\right\}$ are the gradients of these response samples. These gradients can be calculated by solving the deterministic differential equations $\sum_{j=0}^{N} \partial \Psi_{j}$ $\left(X(t ; Z), Y^{(j)}(t)\right) / \partial Z_{i}=0, i=1, \ldots, d$, where $\sum_{j=0}^{N} \Psi_{j}\left(t, X(t), Y^{(j)}(t)\right)=0, t \geq 0$ may be any nonlinear differentiable ODE as described previously, such as the Eqs. (2-5). The gradients $\nabla Y\left(t ; \tilde{z}_{k}\right)$ for the example ODEs are represented by $\partial Y(t ; Z) / \partial Z_{i}$, which are the solutions of Eqs. (2-5) differentiated with respect to each coordinate $Z_{i}$ of $Z$. Explicitly, the gradients $\nabla Y\left(t ; \tilde{z}_{k}\right)$ for the Duffing ODE in Eq. (2) are the solutions of the following equation:

$$
\begin{aligned}
& \frac{\partial \ddot{Y}(t, Z)}{\partial Z_{i}}+2 \zeta v \frac{\partial \dot{Y}(t, Z)}{\partial Z_{i}} \\
& \quad+v^{2} \frac{\partial Y(t, Z)}{\partial Z_{i}}\left(1+3 \rho Y^{2}\left(t ; \tilde{z}_{k}\right)\right)-\varphi_{i}(t)=0 .
\end{aligned}
$$

Note that like Eqs. (2), (15) is also a nonlinear dynamic ODE with unknown $\partial Y(t ; Z) / \partial Z_{i}$, and deterministic input $\varphi_{i}(t), i=1, \ldots, d$. Thus, $d$ deterministic equations must be solved to calculate the gradients $\nabla Y\left(t ; \tilde{z}_{k}\right)$ needed for the construction of the surrogate model in Eq. (14). Similarly, the gradients for the Bouc-Wen ODE in Eq. (3) are calculated by solving the following $d$ equations:

$$
\begin{aligned}
& \frac{\partial \ddot{Y}(t, Z)}{\partial Z_{i}}+2 \zeta \nu \frac{\partial \dot{Y}(t, Z)}{\partial Z_{i}} \\
& +v^{2}\left(\rho \frac{\partial Y(t, Z)}{\partial Z_{i}}+(1-\rho) \frac{\partial W(t, Z)}{\partial Z_{i}}\right) \\
& -\varphi_{i}(t)=0,
\end{aligned}
$$

where

$$
\begin{aligned}
& \frac{\partial W(t, Z)}{\partial Z_{i}}=-\gamma \frac{\partial \dot{Y}(t, Z)}{\partial Z_{i}} \\
& \quad+\alpha\left((n-1) \operatorname{sgn}\left(\tilde{W}_{k}\right)\left|\tilde{W}_{k}\right|^{n-2} \tilde{W}_{k}+\left|\tilde{W}_{k}\right|^{n-1}\right) \\
& \left.\quad \frac{\partial W(t, Z)}{\partial Z_{i}}\right|_{Z=\tilde{z}_{k}}+\beta\left(\frac{\partial \dot{Y}(t, Z)}{\partial Z_{i}}\left|\tilde{W}_{k}\right|^{n}+n \operatorname{sgn}\left(\tilde{W}_{k}\right) \dot{\tilde{Y}}_{k}\left|\tilde{W}_{k}\right|^{n-1}\right. \\
& \left.\left.\quad \frac{\partial W(t, Z)}{\partial Z_{i}}\right|_{Z=\tilde{z}_{k}}\right),
\end{aligned}
$$

$\tilde{W}_{k}=\left(\partial W(t ; Z) / \partial Z_{i}\right) \mid Z=\tilde{z}_{k}$, and $\dot{\tilde{Y}}_{k}=(\partial \dot{Y}(t ; Z)$ $\left./ \partial Z_{i}\right) \mid Z=\tilde{z}_{k}$. Finally, for the NES system defined in Eqs. (4-5), the gradient $\nabla Y\left(t ; \tilde{z}_{k}\right)$ is a bi-dimensional vector with components $\partial Y_{1}(t, Z) / \partial Z_{i}$ and $\partial Y_{2}(t, Z)$ $/ \partial Z_{i}$ calculated by solving the following system of deterministic ODEs:

$$
\begin{aligned}
& \frac{\partial \ddot{Y}_{1}(t, Z)}{\partial Z_{i}}+2 \zeta \nu \frac{\partial \dot{Y}_{1}(t, Z)}{\partial Z_{i}} \\
& +v^{2} \frac{\partial Y_{1}(t, Z)}{\partial Z_{i}}+\lambda_{1}\left(\frac{\partial \dot{Y}_{1}(t, Z)}{\partial Z_{i}}-\frac{\partial \dot{Y}_{2}(t, Z)}{\partial Z_{i}}\right) \\
& \quad+3 c_{1}\left(Y_{1}(t)-Y_{2}(t)\right)^{2}\left(\frac{\partial \dot{Y}_{1}(t, Z)}{\partial Z_{i}}-\frac{\partial \dot{Y}_{2}(t, Z)}{\partial Z_{i}}\right) \\
& \quad+\varphi_{i}(t)=0, \\
& \frac{\partial \ddot{Y}_{2}(t, Z)}{\partial Z_{i}}+\lambda_{2}\left(\frac{\partial \dot{Y}_{2}(t, Z)}{\partial Z_{i}}-\frac{\partial \dot{Y}_{1}(t, Z)}{\partial Z_{i}}\right) \\
& \quad+3 c_{2}\left(Y_{2}(t)-Y_{1}(t)\right)^{2}\left(\frac{\partial \dot{Y}_{2}(t, Z)}{\partial Z_{i}}-\frac{\partial \dot{Y}_{1}(t, Z)}{\partial Z_{i}}\right) \\
& \quad+\varphi_{i}(t)=0 .
\end{aligned}
$$


(a)

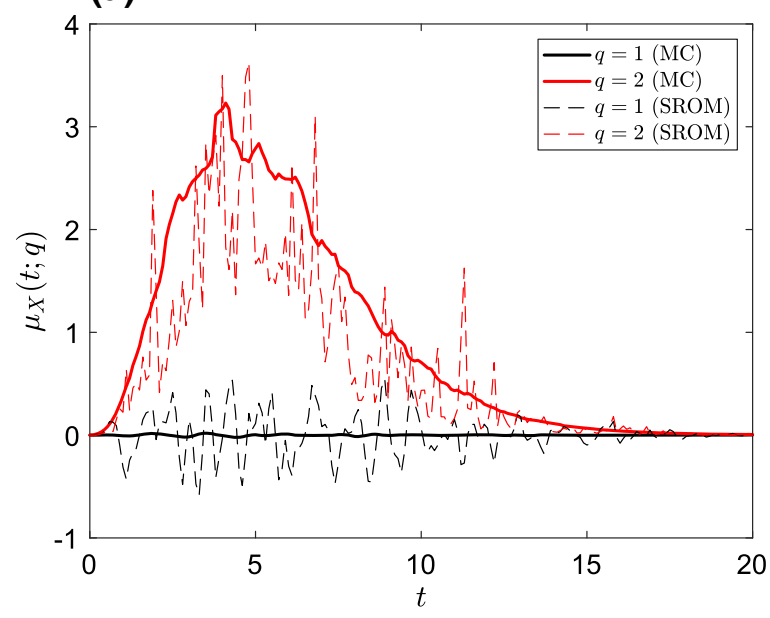

(c)

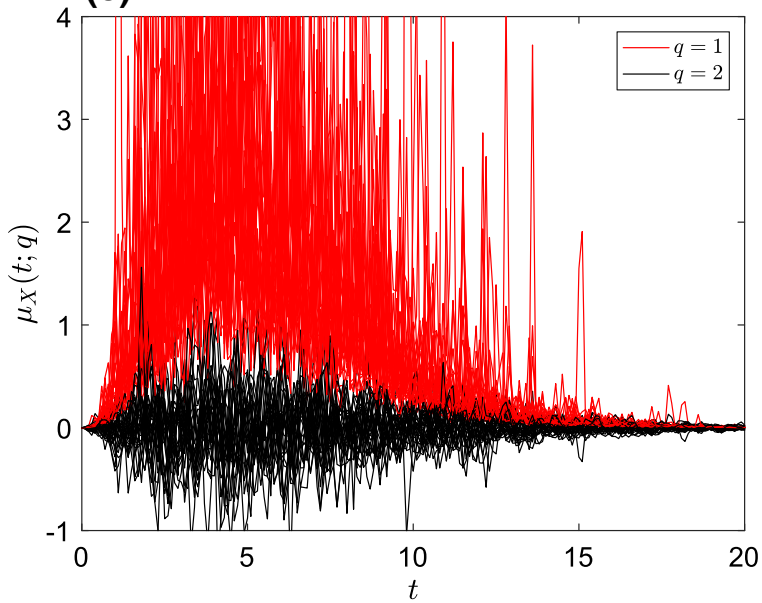

Fig. 2 First two moments $\mu_{X}(t, q), q=1,2$ of $X(t)$ calculated by MC with $n=10^{4}$ samples and the SROM equivalents $\tilde{\mu}_{X}(t, q), q=1,2$ with $\mathbf{a} m=20$ and $\mathbf{b} m=10^{3}$ samples, ver- (b)

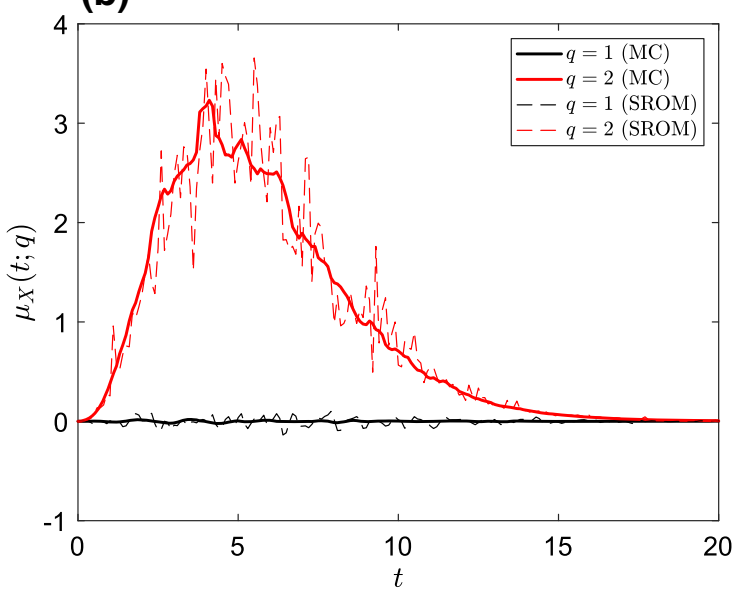

(d)

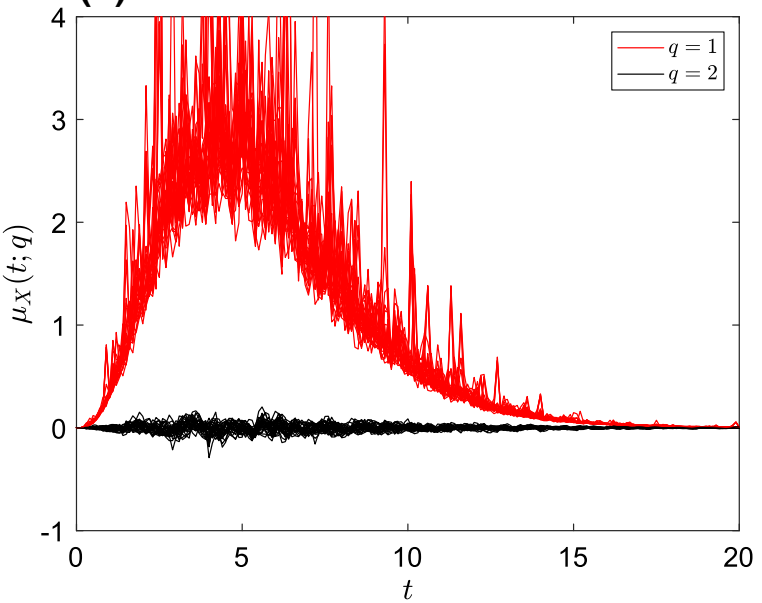

sus 30 trials of them being calculated using MC with $\mathbf{c} n=20$ or $\mathbf{d}=10^{3}$ independent samples of $X(t)$ (a)

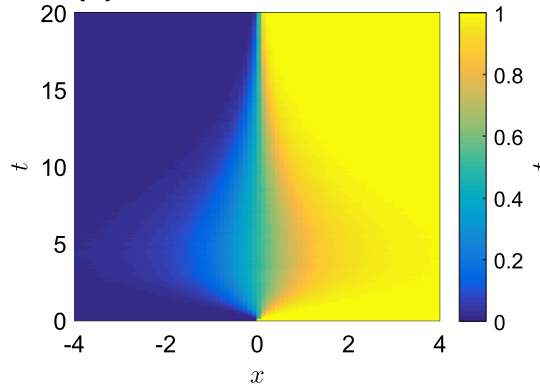

(b)

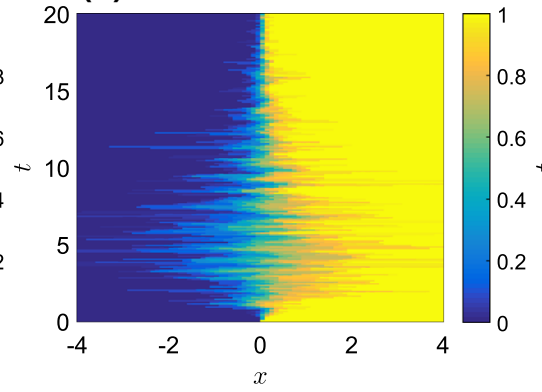

(c)

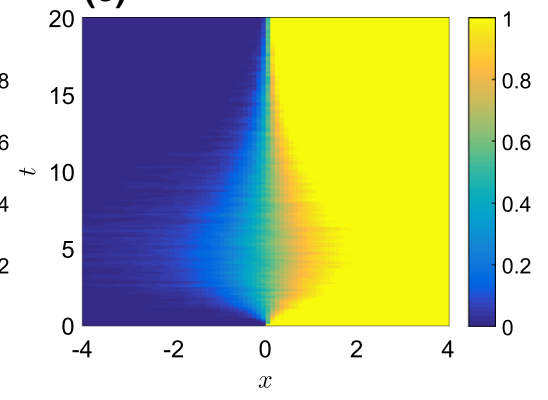

Fig. 3 Marginal distribution functions $F_{X}(x, t)$ of $X(t)$ calculated by a MC with $n=10^{4}$ samples, and the SROM equivalents $\tilde{F}_{X}(x, t)$ of $X(t)$ with $\mathbf{b} m=20$, and $\mathbf{c} m=10^{3}$ samples 
(a)

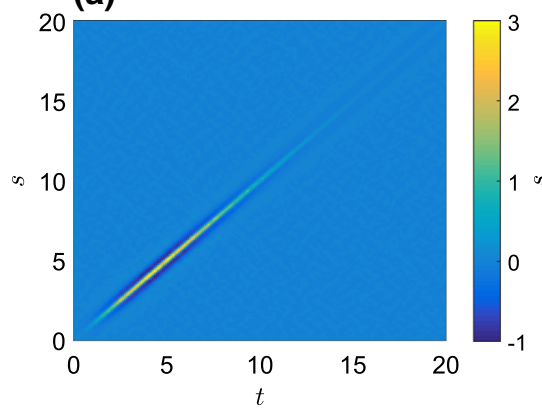

(b)

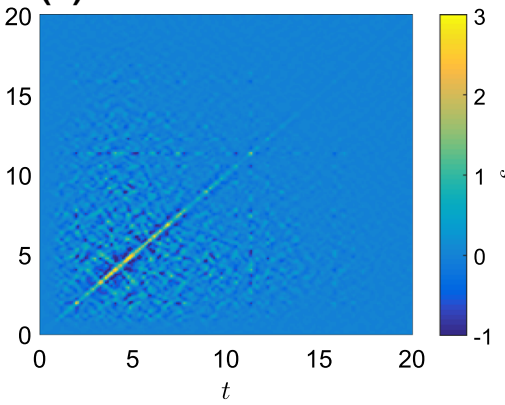

(c)

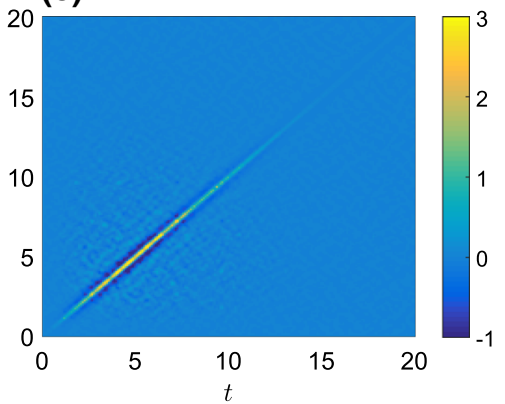

Fig. 4 Covariance functions $\Sigma_{X}(t, s)$ of $X(t)$ calculated by a MC with $n=10^{4}$ samples, and the SROM equivalents $\tilde{\Sigma}_{X}(t, s)$ with $\mathbf{b}$ $m=20$, and $\mathbf{c} m=10^{3}$ samples

Fig. 5 One sample of $X(t)$ (solid, black line) and its parametric approximation (dashes, red line) $\sum_{i=1}^{d} Z_{i} \varphi_{i}(t)$ for $\mathbf{a} d=50$ and $\mathbf{b} d=100$. (Color figure online)
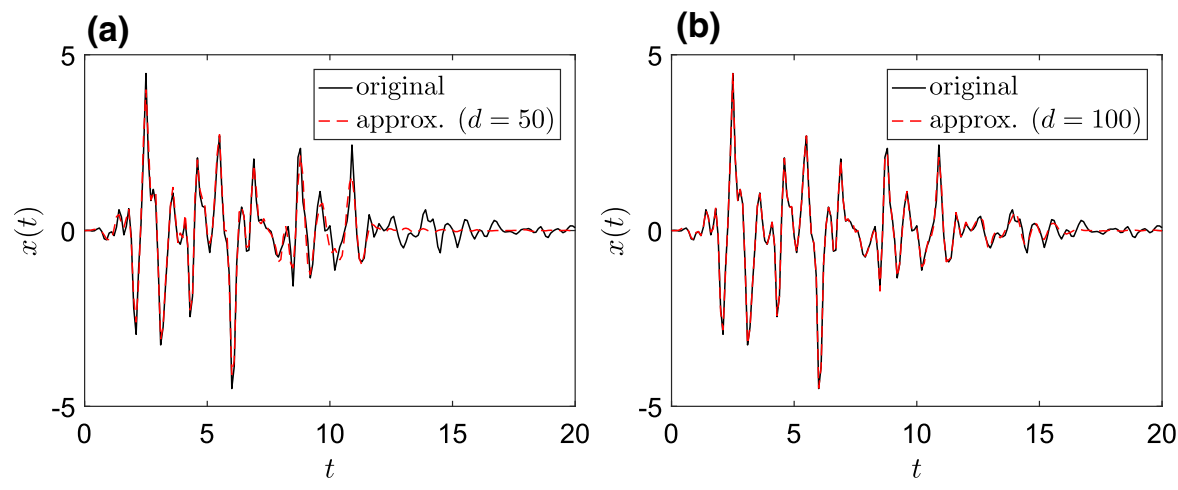

In case of more complex systems, for which explicit ODEs for the gradients cannot be written, they can be approximated numerically from the differences between the responses $Y(t ; Z)$ calculated at $Z=\tilde{z}_{k}$ and $Z=\tilde{z}_{k}+\Delta \tilde{z}_{k}$, where $\Delta \tilde{z}_{k}$ denotes a perturbation of $\tilde{z}_{k}$. Also, higher-order approximations $\tilde{Y}(t ; Z)$ can be constructed and used in the development of the surrogate model in Eq. (14), but they require the calculation of higher-order derivatives of $\tilde{Y}(t ; Z)$ with respect to the coordinates of $Z$. Given that the nonlinear ODEs considered in this study are stable and are differentiable with respect to the systems' states $Y(t)$, the gradients exist, and are solutions of nonlinear ODEs similar to the original ones. Thus, the effort to calculate $\partial Y(t ; Z) / \partial Z_{i}$ is similar to calculating $Y(t)$. Therefore, the number of dynamic analyses needed to construct the surrogate model $\tilde{Y}(t ; Z)$ in Eq. (14) is comprised of $m$ analyses to calculate $Y\left(t ; \tilde{z}_{k}\right)$ at each Voronoi cell centre $\tilde{z}_{k}$, from Eqs. (2-5) and $m \times d$ analyses to calculate $\nabla Y\left(t ; \tilde{z}_{k}\right)\left(Z-\tilde{z}_{k}\right)$ from Eqs. (15-19) for each
Voronoi cell with respect to each coordinate of the random vector $Z$. The major advantage of ESROM is that any number of surrogate response samples $\tilde{y}_{k}(t)$ can be simulated using Eq. (14) in order to increase the accuracy of the response statistics, without any additional computational effort, accounted in the fixed number $m \times(d+1)$ of dynamic analyses needed to construct the surrogate model.

Figure 6 shows the gradients $\partial Y(t ; Z) / \partial Z_{i}$ calculated for the first three coordinates $Z_{i}, i=1,2,3$ of $Z$ for the Voronoi cell centred around the first sample $\tilde{z}_{1}$ of the SROM $\tilde{Z}$, for each of the three nonlinear systems considered. Figure 7 shows the response samples $y(t)$ (black, solid line) calculated by direct integration of Eqs. (2-5) versus the corresponding approximate samples calculated from the surrogate model in Eq. (14) (red, dashed line), for the (a) Duffing, (b) Bouc-Wen and the (c) first and (d) second DOFs of the NES models. 
(a)

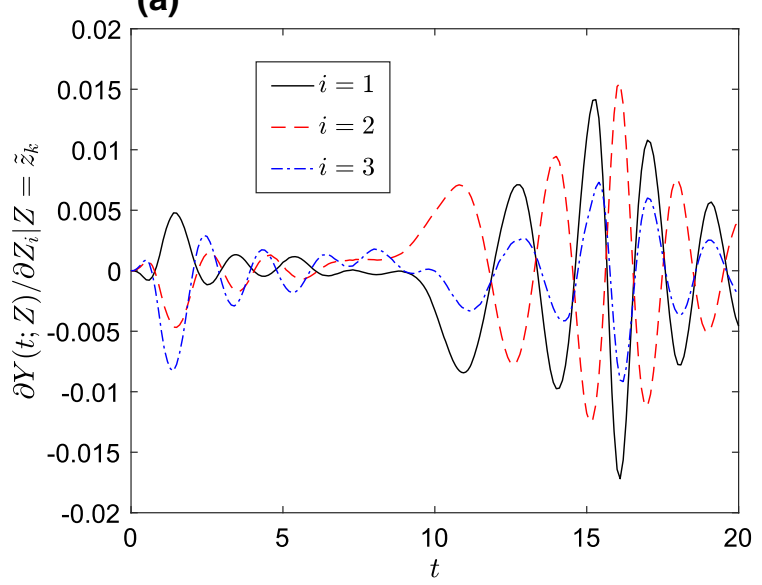

(c)

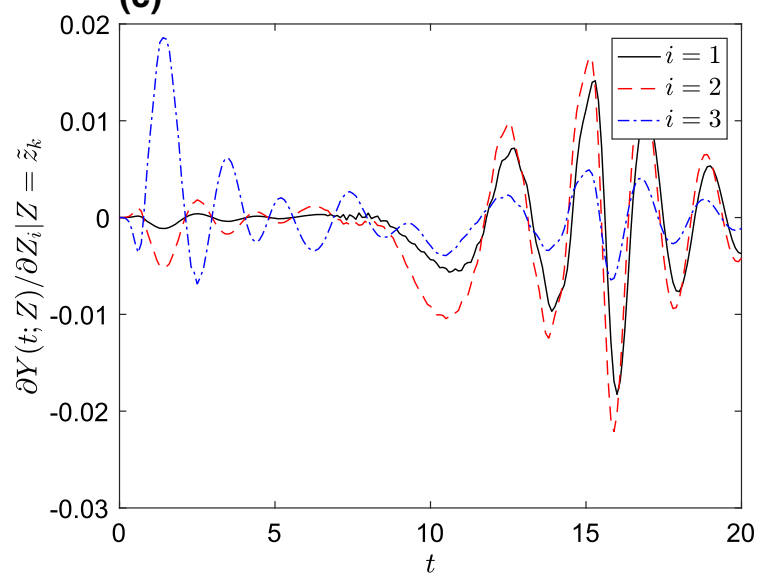

(b)

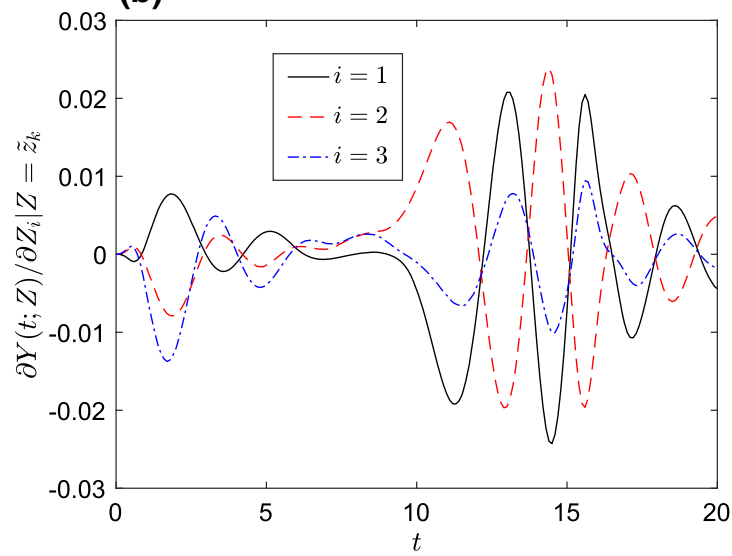

(d)

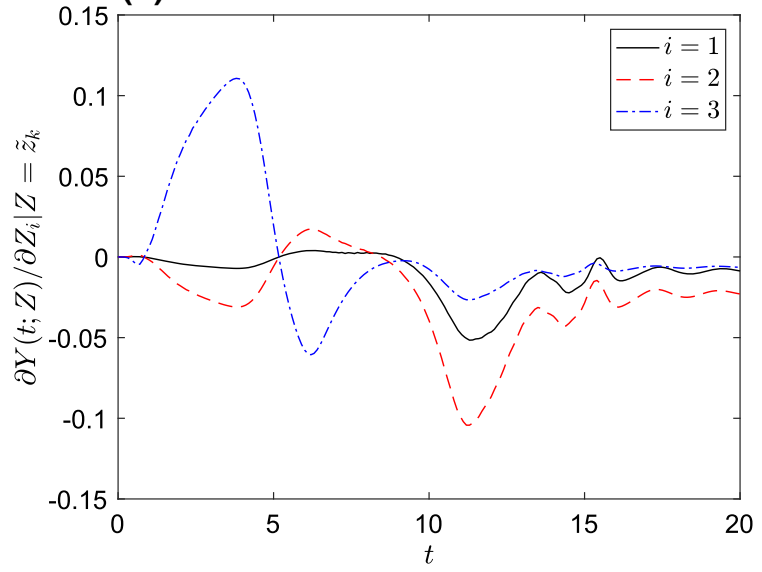

Fig. 6 Gradients $\partial Y(t ; Z) / \partial Z_{i} \mid Z=\tilde{z}_{k}$, for $k=1 ; i=1,2,3$, for the a Duffing, b Bouc-Wen, and $\mathbf{c}$ first and $\mathbf{d}$ second DOFs of the NES system

Example. The following two-dimensional example is considered to illustrate graphically how the ESROM model works. Let's assume that the loading process $X(t)$ is replaced by the bi-variate Gaussian vector $X=\left(X_{1}, X_{2}\right)$, where $X_{1}, X 2 \sim \mathbf{N}(0,1)$ and correlation $\rho_{X_{1}, X_{2}}=0.3$; and the state of the nonlinear system is described by the equation $U(X)=\sin \left(X_{1}\right) \cos \left(X_{2}\right)$. Figure 8a illustrates the first three steps: (i) the green dots are the samples of $x_{k}$ of $X$, (ii) the red stars are the $m=15$ samples $\tilde{x}_{k}$ of the SROM $\tilde{X}$, which are the centres of the (iii) Voronoi cells shown as the black polygons. Figure 8b, c shows the piece-wise linear surrogate model $\tilde{U}(X)$ and the exact solution $U(X)$ (green mesh), respectively. Note that in this simple case, for which the input is a bi-variate random variable rather than a stochastic process, $X$ and $Z$ are identical.
The ESROM estimates of the response statistics defined in Eq. (6) and (7) are

$$
\begin{aligned}
\mathbf{E}\left[Y(t)^{q}\right] & =\frac{1}{n} \sum_{k=1}^{n} \tilde{y}_{k}(t)^{q} \\
F(y) & =\mathbf{P}(Y(t)>y) \\
& =\frac{1}{n} \sum_{k=1}^{n} \mathbf{1}\left(\max _{t \geq 0}\left|\tilde{y}_{k}(t)\right|>y\right),
\end{aligned}
$$

where $\tilde{y}_{k}(t)$ are the samples of $\tilde{Y}(t ; Z)$ calculated in Eq. (14). It is important to note that the response statistics in Eq. (20) and (21) are calculated with response samples from the surrogate model. Thus, the number of samples used for the calculation of the ESROM statis- 
(a)

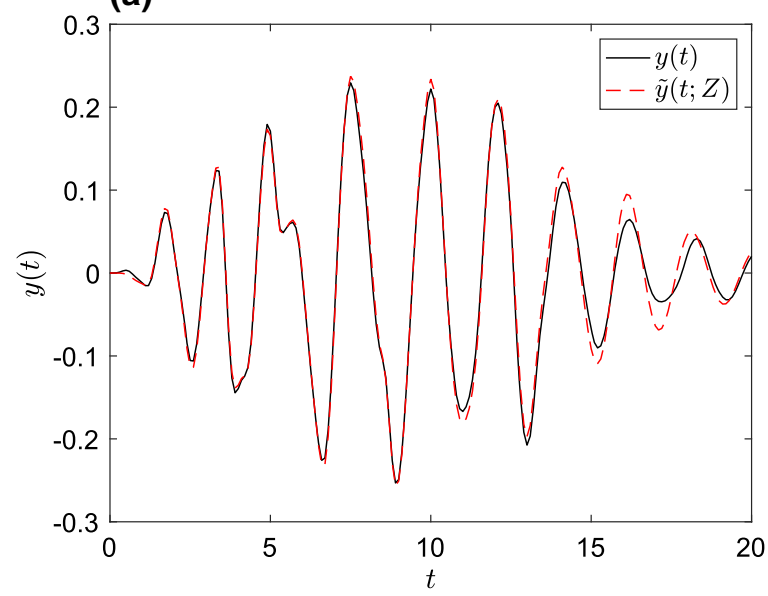

(c)

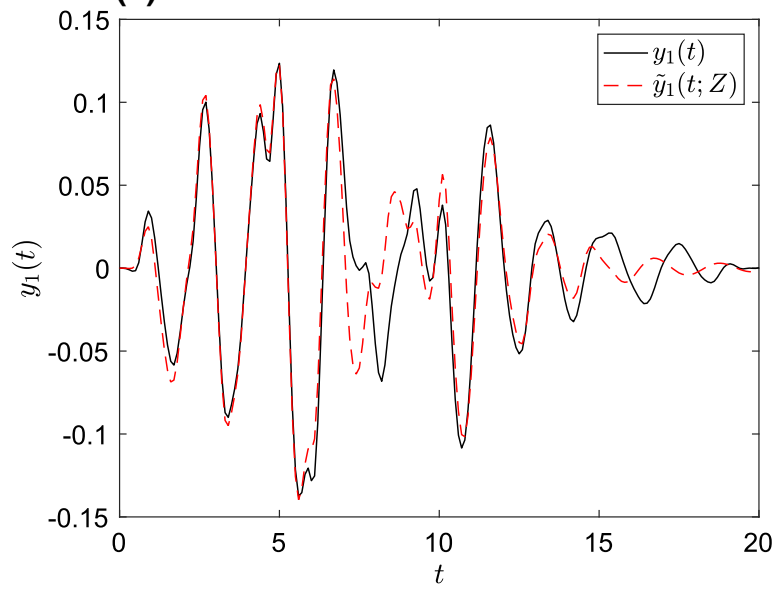

(b)

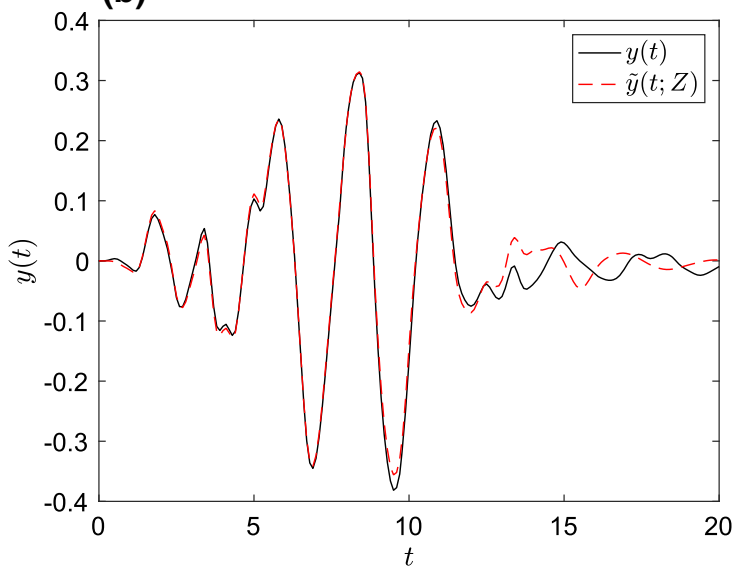

(d)

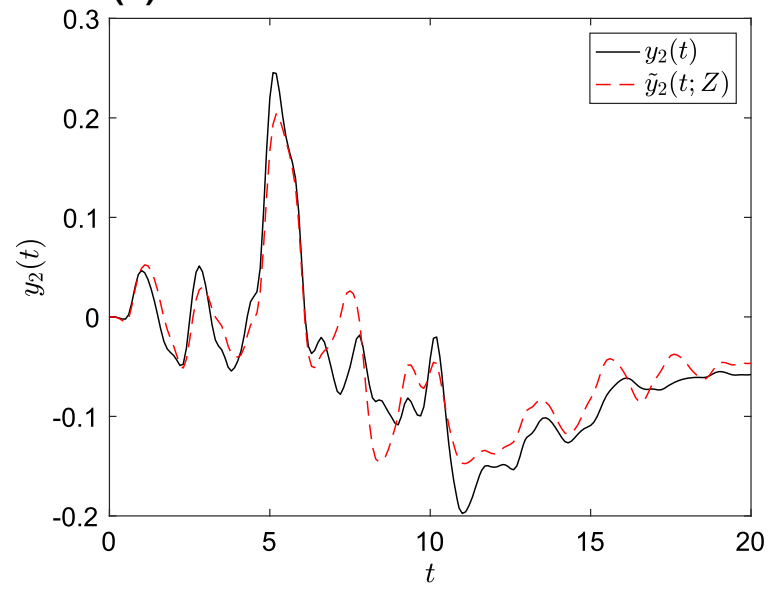

Fig. 7 Response sample of $Y(t)$ (solid, black line) and their corresponding samples from the surrogate model $Y(\tilde{t} ; Z)$ for the a Duffing, b Bouc-Wen, and $\mathbf{c}$ first and $\mathbf{d}$ second DOFs of the NES system

(a)

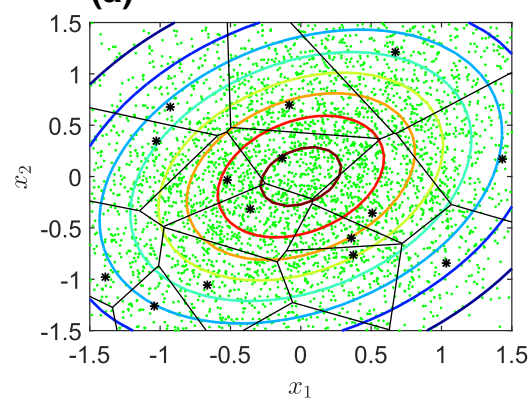

(b)

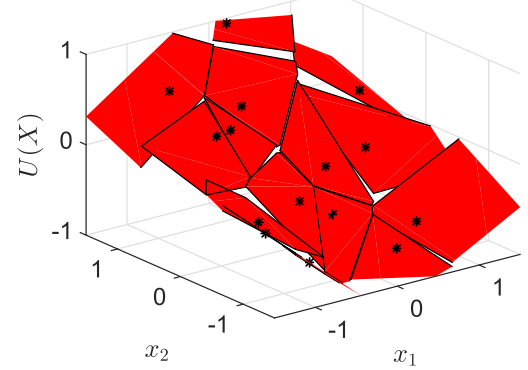

(c)

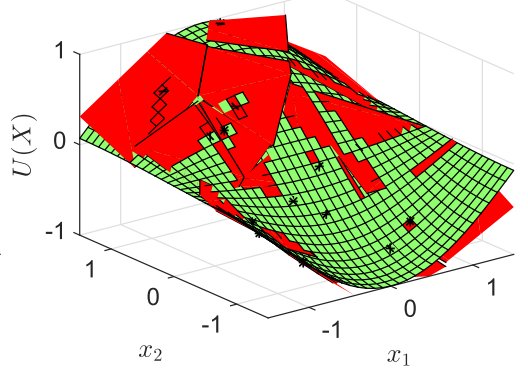

Fig. 8 Example. a SROM $\tilde{X}$ for $X$, b piece-wise linear surrogate solution $\tilde{U}(G)$, for $m=15$, and c exact solution $U(X)=$ $\sin \left(X_{1}\right) \cos \left(X_{2}\right)$. (Color figure online) 
(a)

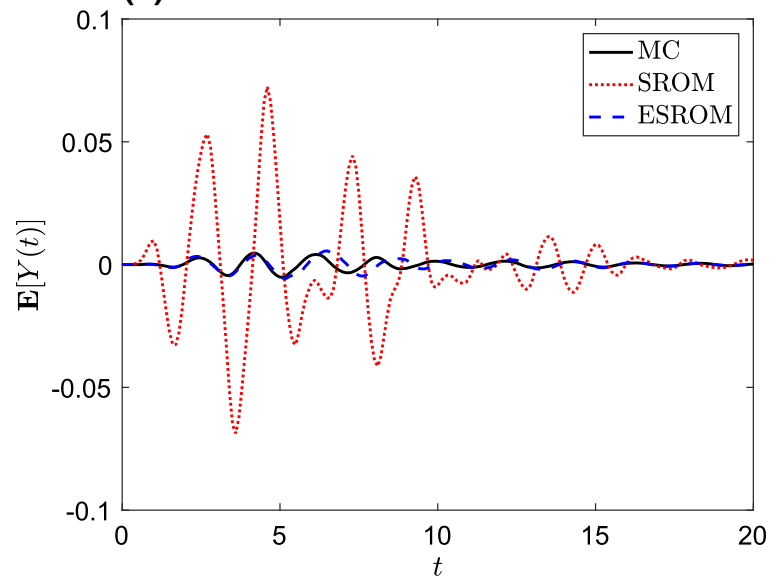

(c)

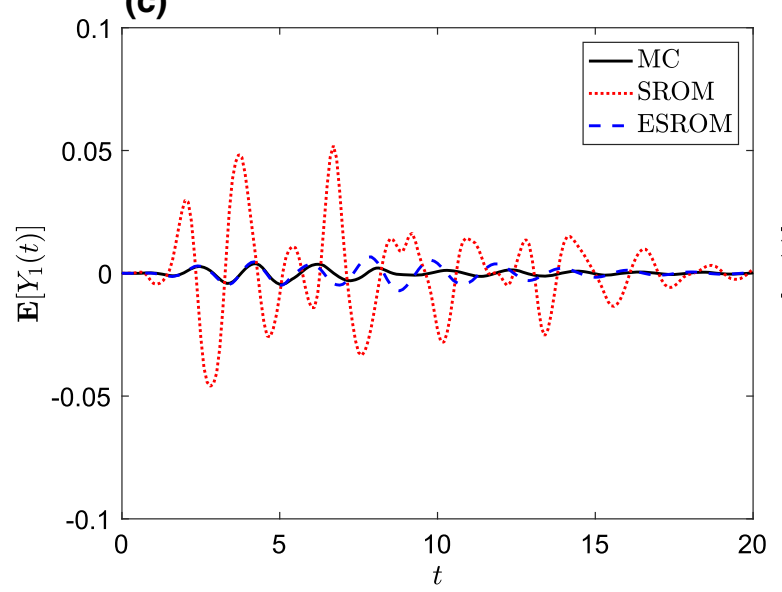

Fig. 9 First-order moments $\mathbf{E}[Y(t)]$ using MC with $n=10^{4}$ (black, solid line), SROM with $m=20$ (red, dotted line), and ESROM with $d=100, m=10$ (blue, dashed line), for the a

tics is not resumed to just $m$, but can go up to $n$, the total number of samples of $X(t)$ available, since each sample $\tilde{y}_{k}(t)$ is calculated directly from Eq. (14) using the sample of $Z$ corresponding to each sample of $X(t)$, as per the approximation in Eq. (13), without performing any additional dynamic analyses.

\section{Numerical results and discussion}

The performance of the two methods proposed in the previous sections is discussed in terms of the accuracy with which they are able to estimate statistics such as the moments or the tail probability distributions for the three nonlinear dynamic systems consid- (b)

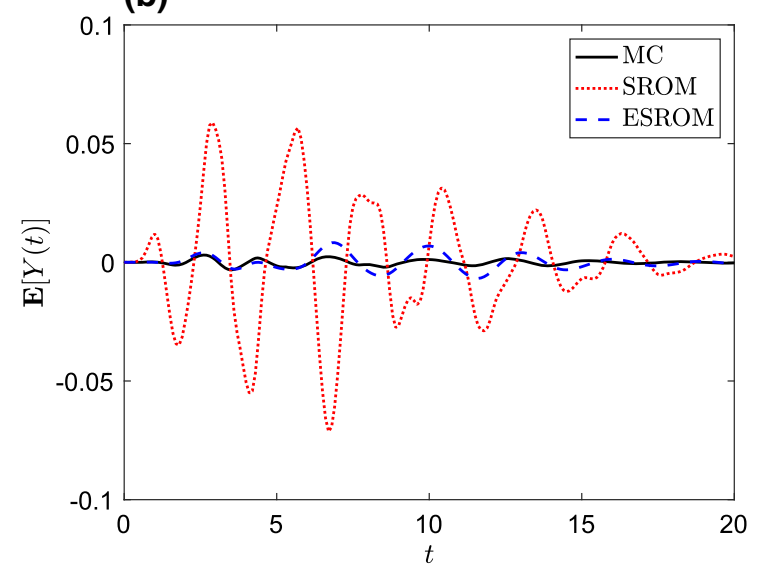

(d)

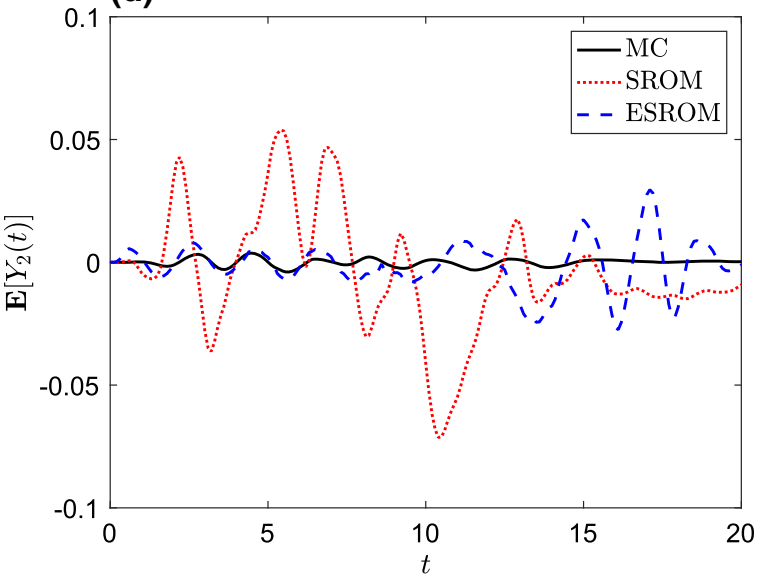

Duffing, $\mathbf{b}$ Bouc-Wen, and $\mathbf{c}$ first and $\mathbf{d}$ second DOFs of the NES system. (Color figure online)

ered, with respect to the MC estimates of the same results. Figures 9 and 10 show estimates of the firstand second-order moments $\mu(t, q=1)=\mathbf{E}[Y(t)]$ and $\mu(t, q=2)=\mathbf{E}\left[Y(t)^{2}\right]$, respectively, for the nonlinear systems' responses, by using the SROM-estimates in Eq. (11) with $m=20$ (red, dotted line); the ESROM estimates in Eq. (20) with $d=100, m=10$ (blue, dashed line), in comparison with the MC estimates in Eq. (6) with $n=10^{4}$ (black, solid line), for each of the three systems chosen for our examples, i.e. the (a) Duffing, (b) Bouc-Wen and the (c) first and (d) second DOFs of the NES systems.

Using the same number of samples as in Figs. 9 and 10, Fig. 11 shows the tail probability distribution estimates $F(y)=\mathbf{P}\left[\max _{t \geq 0}|Y(t)|>y\right]$ of the non- 
(a)

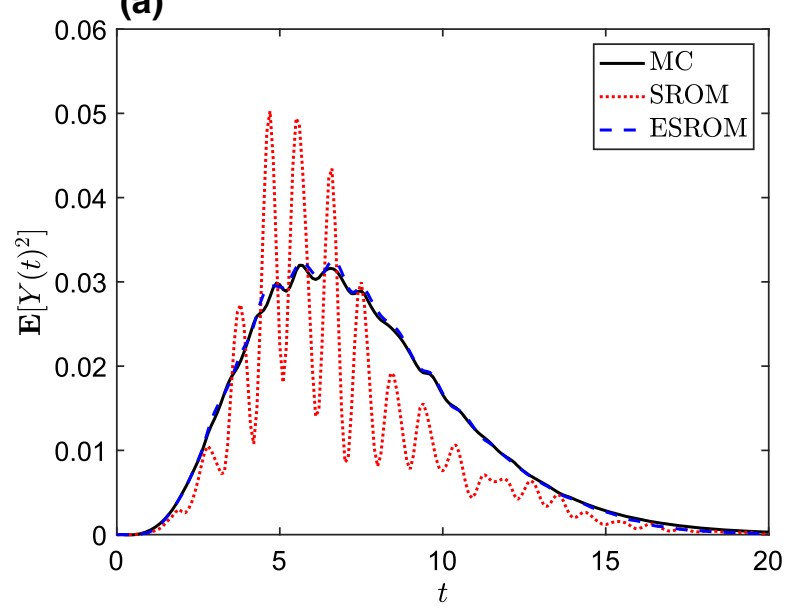

(c)

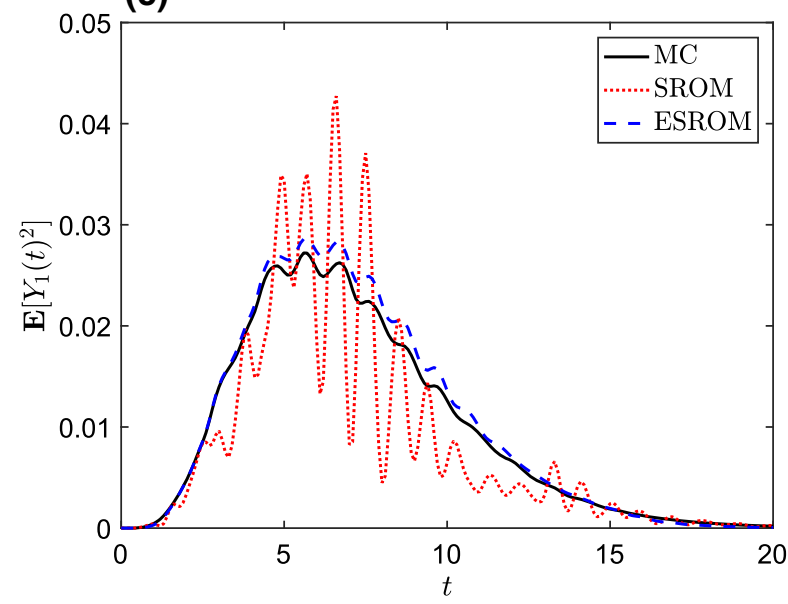

Fig. 10 Second-order moments $\mathbf{E}\left[Y(t)^{2}\right]$ using MC with $n=$ $10^{4}$ (black, solid line), SROM with $m=20$ (red, dotted line), and ESROM with $d=100, m=10$ (blue, dashed line), for the

linear systems' responses, by using the three SROM (red, dotted line), ESROM (blue, dashed line) and the MC (black, solid line), for the same three nonlinear systems.

The accuracy and the efficiency of the SROM-based methods proposed depend on the SROM and ESROM models' sizes. For example, the accuracy of the SROM $\tilde{X}(t)$ depends on the model size $m$, but also for a selected $m$ it depends also on the optimization algorithm used to select its samples $\tilde{x}(t)$ and their probabilities $p_{k}$. It was shown in [41] that the SROM solution converges to the MC solution as $m \rightarrow \infty$. Thus, a large dimension $m$ of the SROM would produce very accurate statistics of the process $X(t)$, but it comes at a large (b)

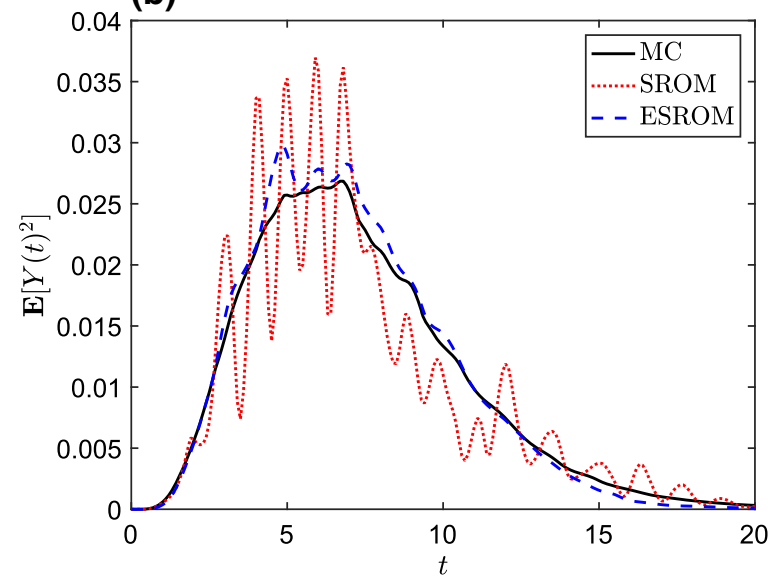

(d)

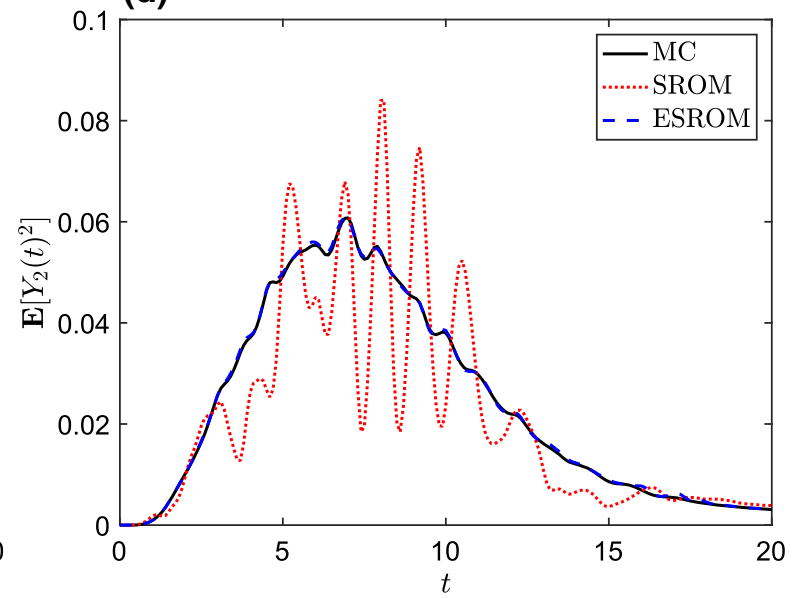

a Duffing, b Bouc-Wen, and $\mathbf{c}$ first and $\mathbf{d}$ second DOFs of the NES system. (Color figure online)

computational cost, similar to Monte Carlo [35]. Even though accurate response statistics have been achieved with low values of $m[40,41]$, the size $m$ must be chosen on consideration of computational time. The accuracy of the response statistics for $Y(t)$ depends on that of $\tilde{X}(t)$ and the approximation used for the mapping of $X(t)$ into $Y(t)$. Bounds for the errors of SROM-based methods have been established in [59] and [38], but this analysis is beyond the scope of this paper.

Figures 12 and 13 show the same first- and secondorder-moment estimates, similar to Figs. 9 and 10, but with an increased size of the SROM $\tilde{X}(t)$ in the SROM method to $m=10^{3}$, which is similar to the computational effort required to build the response sur- 
(a)

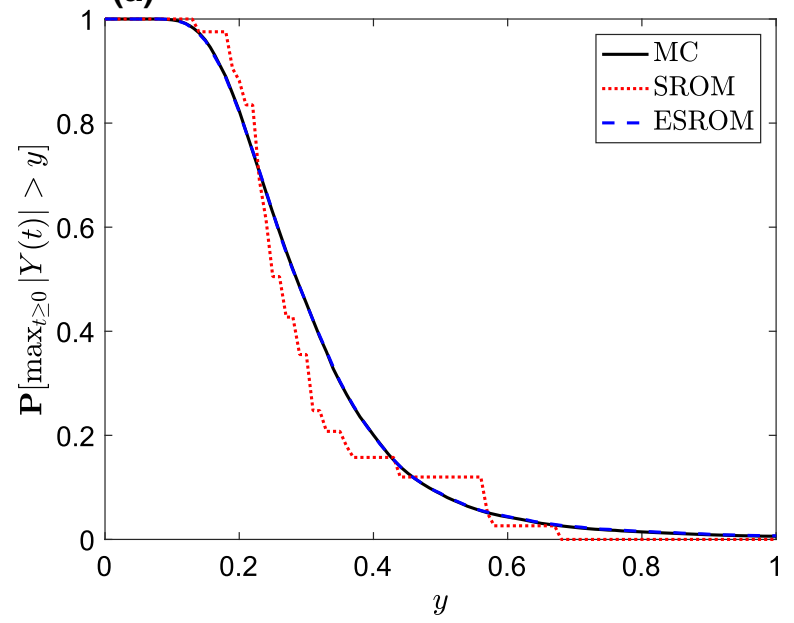

(c)

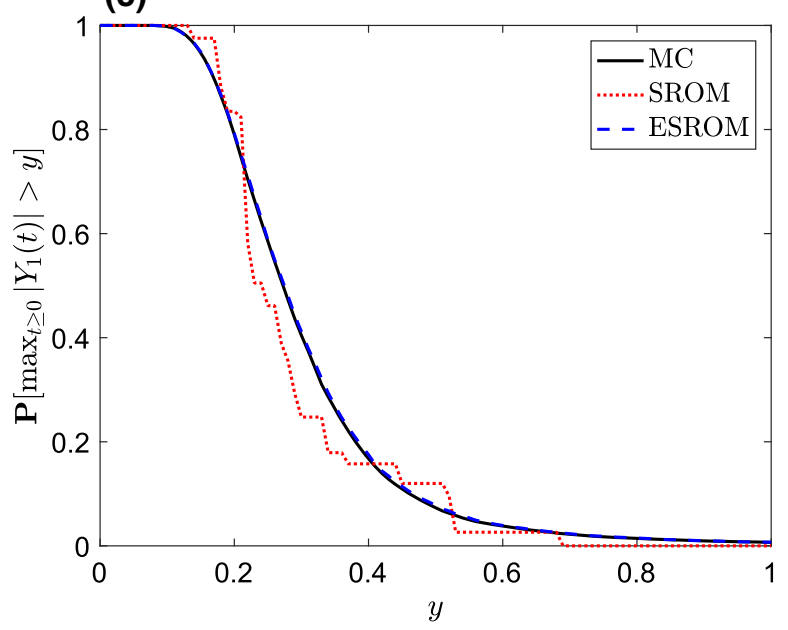

Fig. 11 Tail distribution $\mathbf{P}\left[\max _{t \geq 0}|Y(t)|>y\right]$ using MC with $n=10^{4}$ (black, solid line), SROM with $m=20$ (red, dotted line), and ESROM with $d=100, m=10$ (blue, dashed line),

rogate model in the ESROM method. As expected, the increase in $m$ provides improved results of the SROM method, similar to the ones obtained by MC and ESROM.

While in the SROM method the estimates of the response statistics are based on a rather crude approximation of the mapping between the response samples $\tilde{y}_{k}(t)$ and the SROM samples $\tilde{x}_{k}(t)$, the ESROM method is based on a more accurate representation of this mapping, as shown in the previous section. The accuracy of the ESROM method depends as well on the dimension $m$ of the SROM model $\tilde{Z}$ of $Z$, but also on the dimension $d$ of the vector $Z$, which is used to parametrize the input process $X(t)$. The effi- (b)

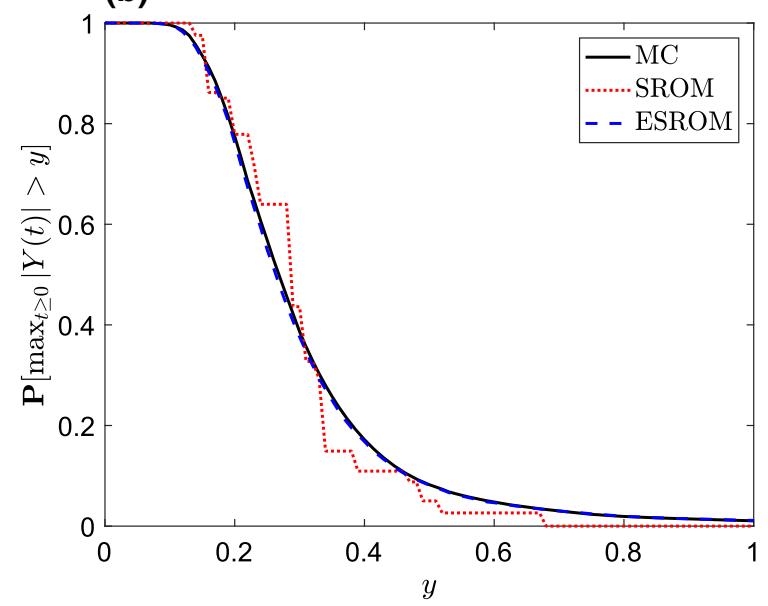

(d)

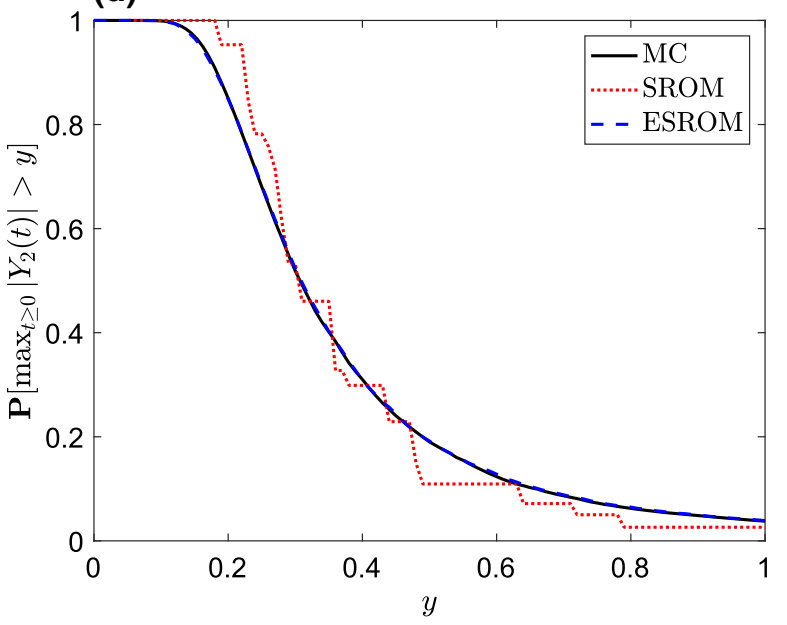

for the a Duffing, b Bouc-Wen, and $\mathbf{c}$ first and $\mathbf{d}$ second DOFs of the NES system. (Color figure online)

ciency of the methods proposed will be judged by the number of deterministic nonlinear dynamic analyses involved to calculate the response statistics. While MC requires $n$ dynamic analyses, the SROM requires only $m<<$ dynamic analyses for the samples $\left(\tilde{x}_{k}(t), p_{k}\right), k=1, \ldots, m$ of the SROM $X(t)$. The ESROM method requires a larger number of dynamic analyses than SROM, that is, $m \times(d+1)$ analyses. As a result, the efficiency of the ESROM method depends on the balance between the dimension $d$ of $Z$ and the dimension $m$ of its SROM $\tilde{Z}$. In order to get a sense of how the balance between $m$ and $d$ plays a role in the development of the ESROM method, Fig. 14a shows the tail distributions for the response of the Bouc- 
(a)

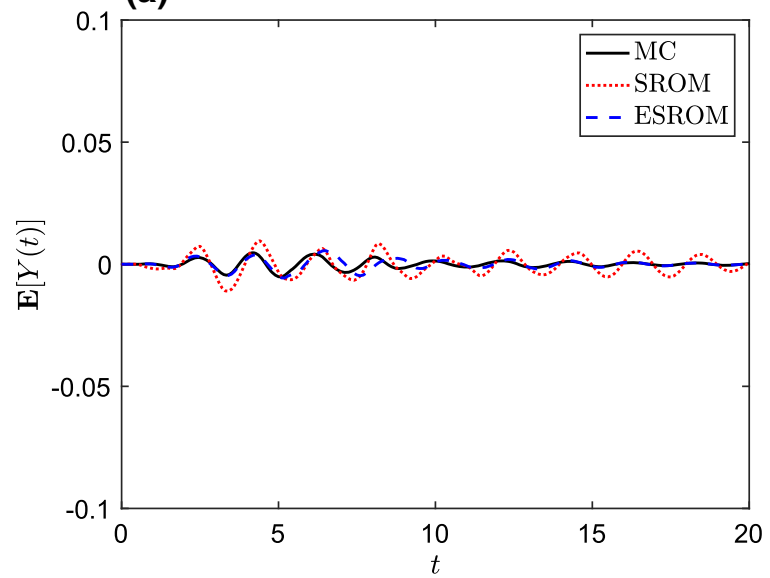

(c)

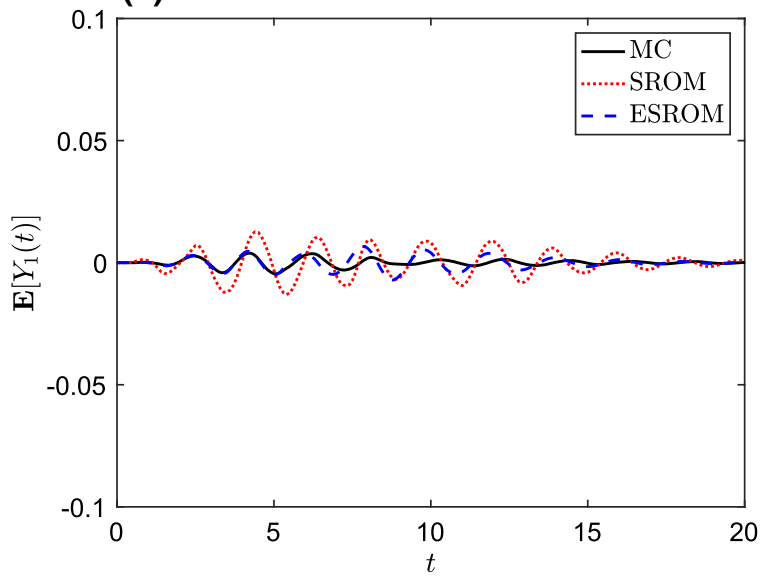

Fig. 12 First-order moments $\mathbf{E}[Y(t)]$ using MC with $n=10^{4}$ (black, solid line), SROM with $m=10^{3}$ (red, dotted line), and ESROM with $d=100, m=10$ (blue, dashed line), for the a

Wen model for approximately the same computational effort, i.e. approximately $10^{3}$ dynamic analyses, but for two sets of $m$ and $d$, i.e. $m=20, d=50$ and $m=10, d=100$, respectively. It is noticed that an increase in the size $m$ of the $\tilde{Z}$ at the expense of the dimension $d$ of $Z$ leads to results that are divergent from the MC solution. Thus, the accurate representation of $X(t)$ in Eq. (13), which is controlled by the dimension of $Z$ (see Fig. 5), is essential for the success of the method. This conclusion is in line with expectation since the higher the dimension $d$ is, the better the frequency content of $X(t)$ is modelled, and the response of nonlinear dynamic systems is sensitive to the frequency content of the excitation. Figure $14 \mathrm{~b}$ shows that (b)

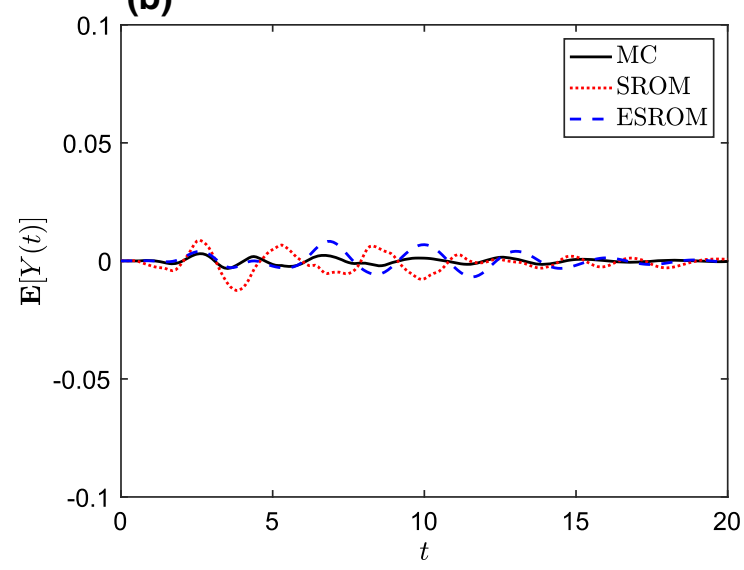

(d)

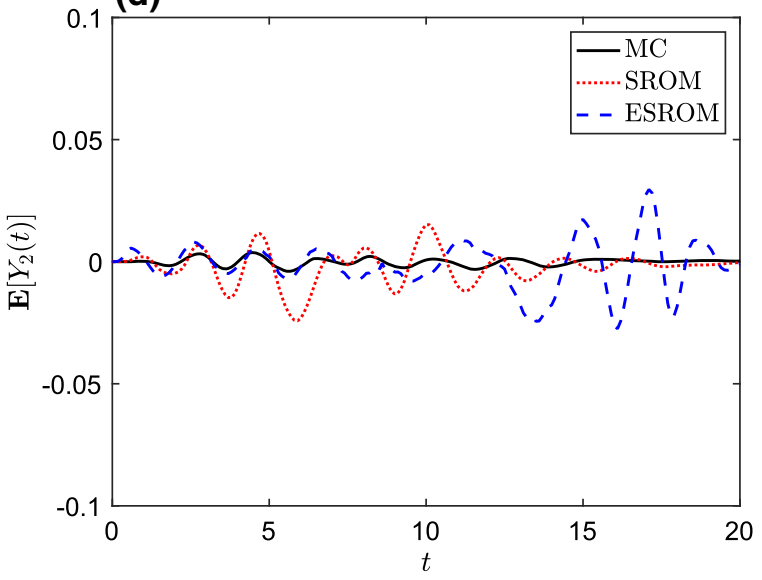

Duffing, b Bouc-Wen, and $\mathbf{c}$ first and $\mathbf{d}$ second DOFs of the NES system. (Color figure online)

an increase in the dimension $m$ in the SROM method, provides better results also for the tail distribution of the response of the Bouc-Wen system, as already seen for the moments in Figs. 12 and 13.

Further plots and discussions on the accuracy and efficiency of the two methods proposed for the tail distributions of the response of the other two systems are shown in Figs. 15 and 16. Figure 15 shows the response tail distributions in Fig. 11 in a logarithmic scale including for extreme values of the response, i.e. $y>1$. It can be seen that the solutions provided by MC $\left(n=10^{4}\right)$, SROM $(m=20)$ and $\operatorname{ESROM}(m=10, d=100)$ diverge as $y$ increases. 
(a)

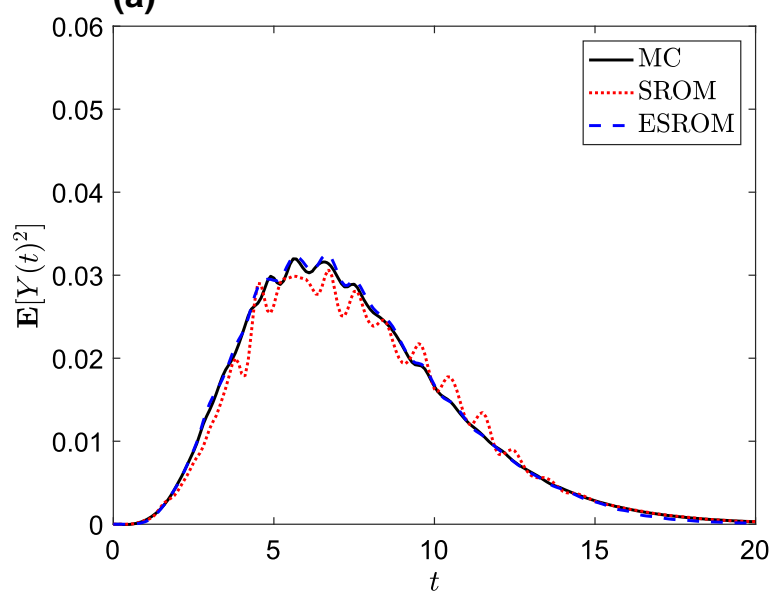

(c)

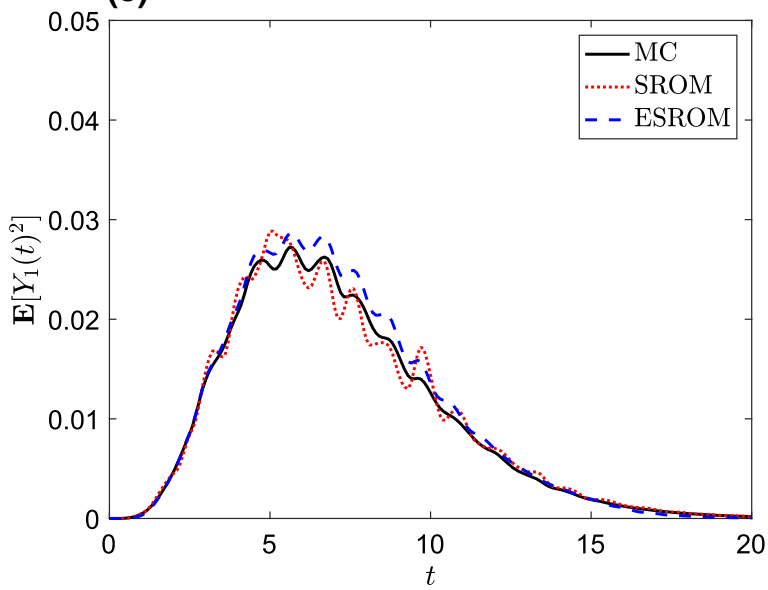

Fig. 13 Second-order moments $\mathbf{E}\left[Y(t)^{2}\right]$ using MC with $n=$ $10^{4}$ (black, solid line), SROM with $m=10^{3}$ (red, dotted line), and ESROM with $d=100, m=10$ (blue, dashed line), for the (b)

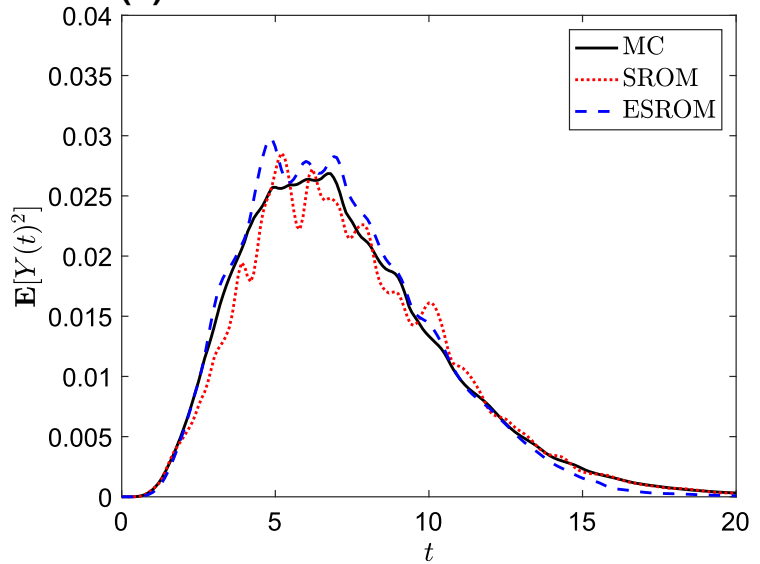

(d)

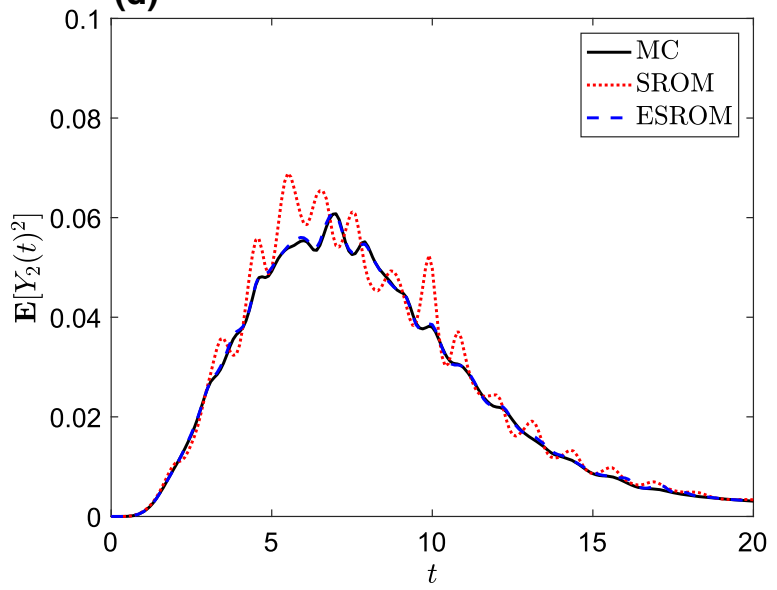

a Duffing, b Bouc-Wen, and $\mathbf{c}$ first and $\mathbf{d}$ second DOFs of the NES system. (Color figure online)
Fig. 14 Tail distribution $\mathbf{P}\left[\max _{t \geq 0}|Y(t)|>y\right]$ using MC with $n=10^{4}$ (black, solid line) and a ESROM with $d=100, m=10$ (blue, dashed line), ESROM with $d=50, m=20$ (blue, dot-dashed line); b SROM with $m=20$ (red, dotted line), SROM with $m=10^{3}$ (red, dot-dashed line) for the Bouc-Wen systems. (Color figure online) (a)

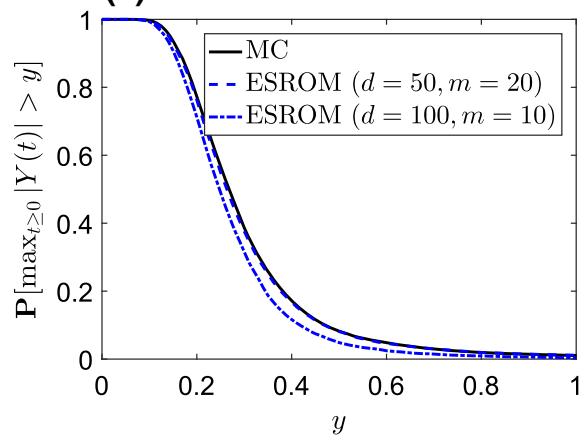

(b)

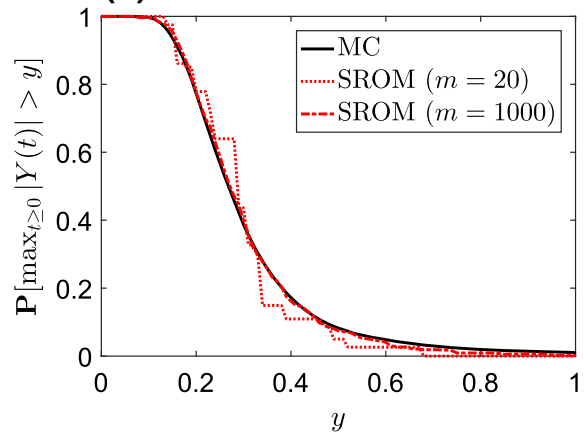


(a)

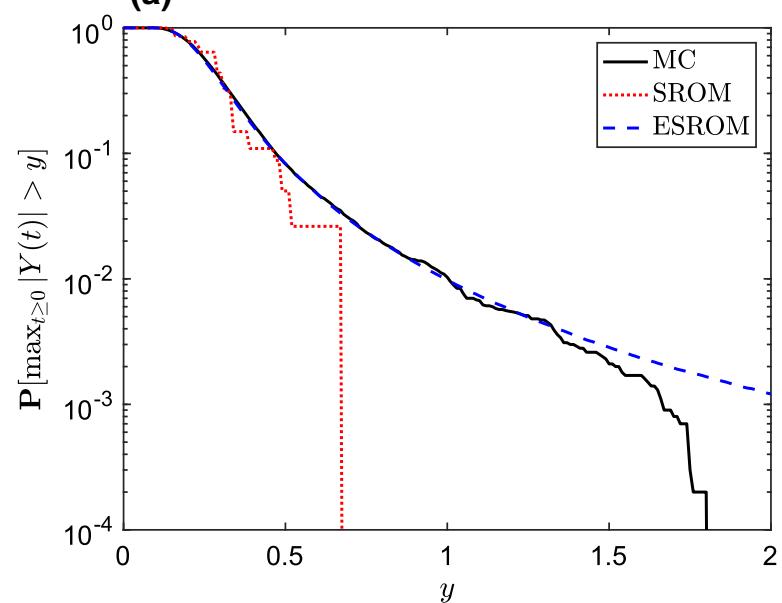

(c)

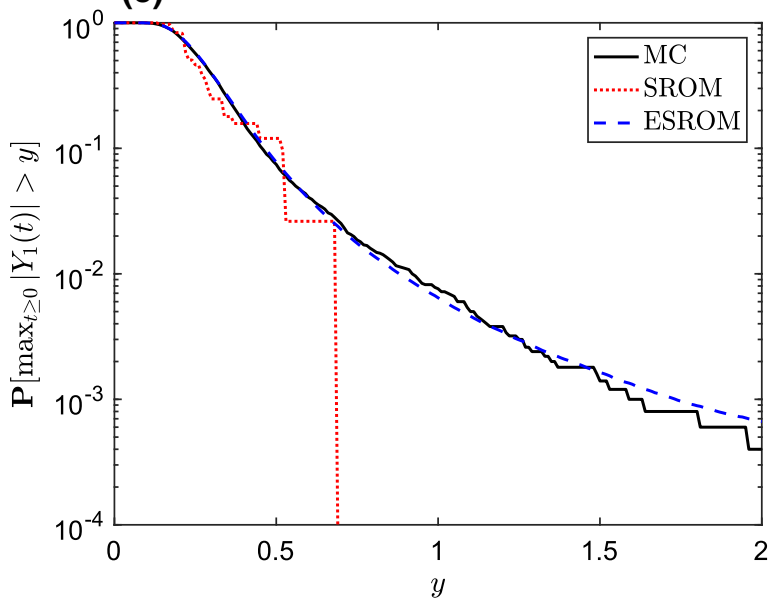

Fig. 15 Extreme tail distribution $\mathbf{P}\left[\max _{t>0}|Y(t)|>y\right]$ using MC with $n=10^{4}$ (black, solid line), SROM with $m=20$ (red, dotted line), and ESROM with $d=100, m=10$ (blue, dashed

Figure 16 shows the same results as in Fig. 15, but for a larger number of MC samples, increased from $n=10^{4}$ to $n=10^{6}$, and for a larger dimension $m$ in the SROM method from $m=20$ to $m=10^{3}$. A very large number of samples $\left(n=10^{6}\right)$ are required for the MC method to reach convergence in the extreme tails for values of $y>1$. Regarding the SROM method, we notice that a large size $m=10^{3}$ is still insufficient to improve results in the extreme tails. However, the ESROM results for the initial values $m=10, d=100$ provide excellent results, similar to the MC results calculated for $n=10^{6}$. Thus, with only approximately one thousandths of the computational effort, the ESROM is able to provide accurate results even (b)

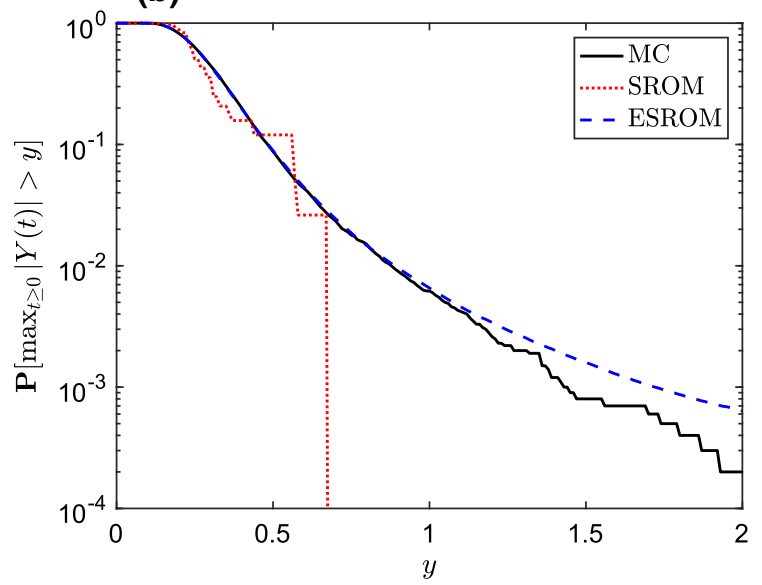

(d)

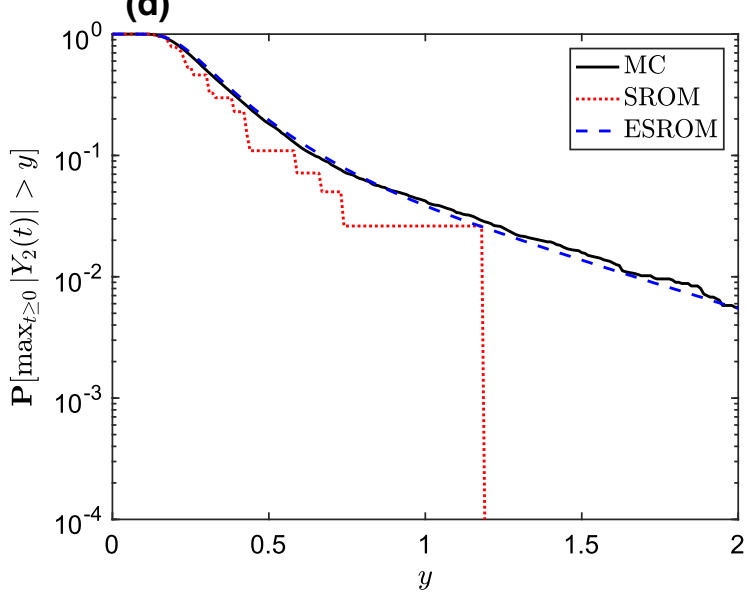

line), for the a Duffing, b Bouc-Wen, and $\mathbf{c}$ first and $\mathbf{d}$ second DOFs of the NES system. (Color figure online)

in the tail of the response of the distribution of $Y(t)$. Nevertheless, the SROM is a very efficient and reliable alternative to $\mathrm{MC}$ for calculating the other response statistics, at just a very small fraction of the computational effort.

Indisputable advantages of the ESROM method are shown in the analyses of extreme values of the state $Y(t)$ of the nonlinear systems. In order to analyse the extreme values of $Y(t)$, Fig. 15 shows the tail distributions for the Duffing and the Bouc-Wen models in a logarithmic scale. In the case of extreme-value analysis, by comparing the Figs. 15 and 16, we can conclude that the ESROM clearly outperforms MC method, managing to produce reliable results for just a small frac- 
(a)

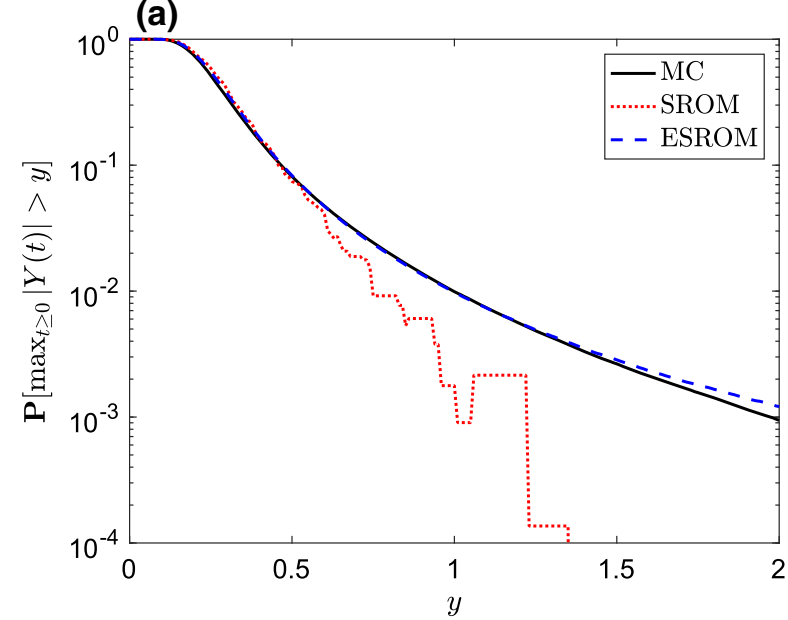

(c)

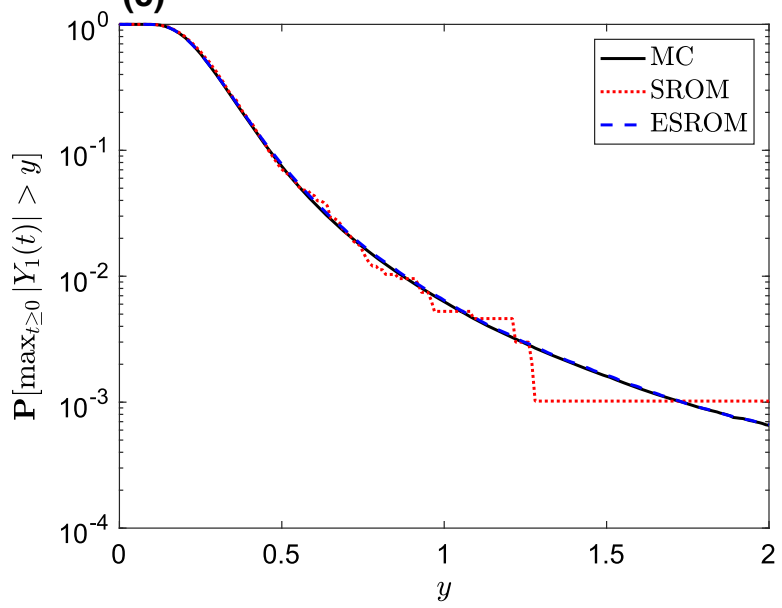

Fig. 16 Extreme tail distribution $\mathbf{P}\left[\max _{t \geq 0}|Y(t)|>y\right]$ using MC with $n=10^{6}$ (black, solid line), SROM with $m=10^{3}$ (red, dotted line), and ESROM with $d=100, m=10$ (blue, dashed

tion of the deterministic analyses required by the MC method to reach convergence. The two SROM-based methods proposed are good alternatives to MC and provide accurate results efficiently, which allows for simulation-based results to be estimated accurately for nonlinear systems subjected to stochastic input. Finally, given the superior performance of the ESROM solution compared with MC throughout the distribution of the response, even in the case of extreme values, any statistics can be calculated with limited effort. To support this idea, a more practical example of the joint distributions of the maxima of the input $X(t)$ and the response $Y(t)$ for the NES system is shown in Fig. 17. Since both processes $X(t)$ and $Y(t)$ are (b)

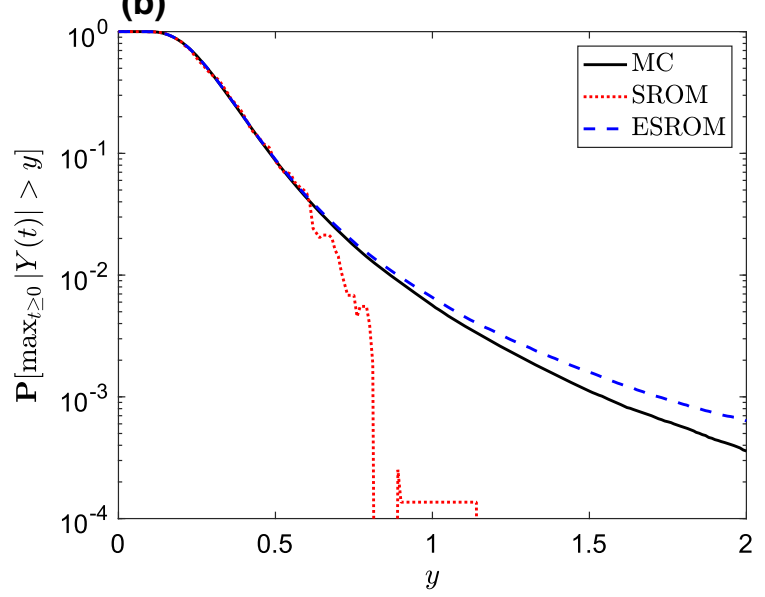

(d)

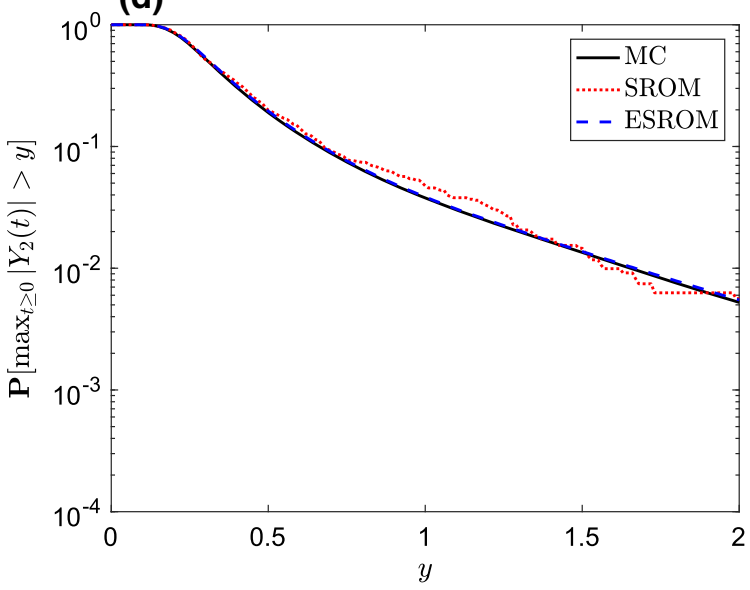

line), for the a Duffing, b Bouc-Wen, and $\mathbf{c}$ first and $\mathbf{d}$ second DOFs of the NES system. (Color figure online)

non-stationary, their joint distribution is impractical to show and thus the conditional probabilities of the maxima $\mathbf{P}\left[\max _{t \geq 0}\left|Y_{j}(t)\right|>y\left|\max _{t \geq 0}\right| X(t) \mid>x\right]$ for each of the 2DOFs $j=1,2$ of the NES system are calculated and shown in Fig. 17a-d, calculated using the MC and the ESROM responses, respectively. The SROM results for this statistic are not shown since this method fails to perform accurately in case of large values of the response $Y(t)$, and it does so for the joint distribution of the maxima of $X(t)$ since it would be restricted to evaluate the maxima only through the small $m$ number of input samples used, insufficient for such a result. 

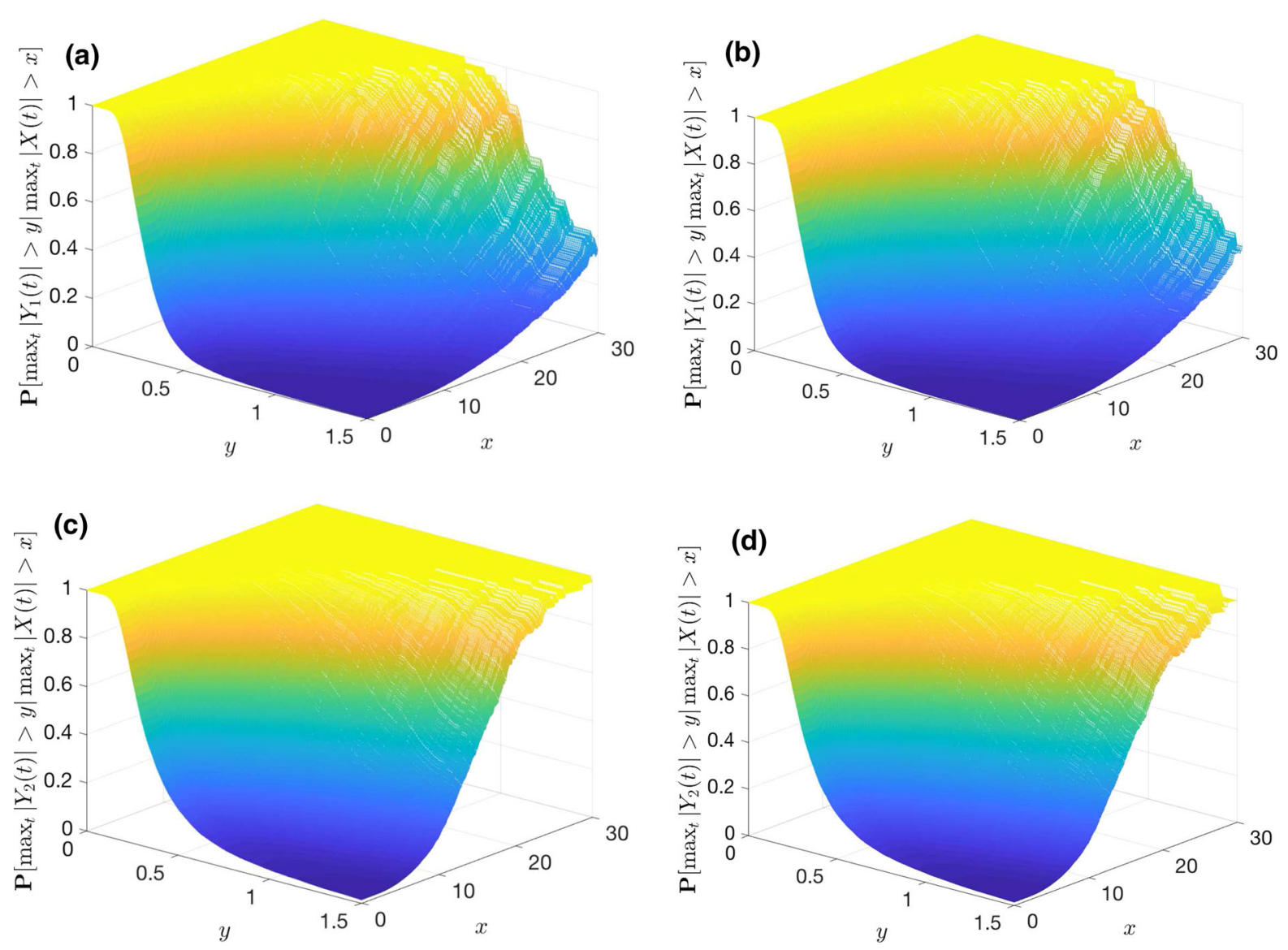

Fig. 17 Conditional probability of exceedance for: the first DOF of the NES system $\mathbf{P}\left[\max _{t \geq 0}\left|Y_{1}(t)\right|>y\left|\max _{t \geq 0}\right| X(t) \mid>x\right]$ calculated using $\mathbf{a}$ MC and $\mathbf{b}$ ESROM; and the second DOF of the

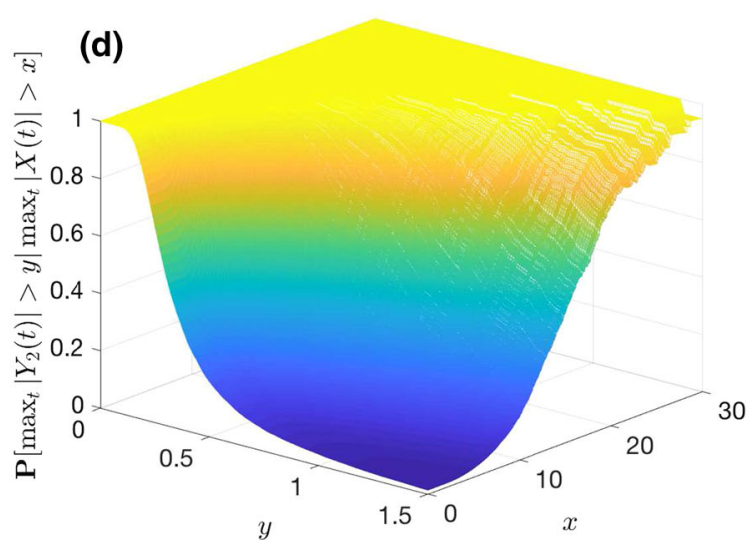

NES system $\mathbf{P}\left[\max _{t \geq 0}\left|Y_{2}(t)\right|>y\left|\max _{t \geq 0}\right| X(t) \mid>x\right]$ calculated using $\mathbf{c}$ MC and $\mathbf{d}$ ESROM, with $n=10^{6}, d=100, m=$ 10

\section{Conclusions}

Two general, efficient and reliable methods are proposed for solving stochastic stable nonlinear dynamic equations. Stochastic reduced-order models (SROM), that is, random processes with finite number of samples, have been used to represent the stochastic inputs to nonlinear dynamic systems and construct approximations for the states of these systems. Two types of SROM-based solutions were presented, and numerical results were compared with the Monte Carlo (MC) estimates. The first method, that is, the SROM-based solution involves the construction of a SROM for the input process, whose samples are used to calculate the responses of the nonlinear systems. The extended version of this method, that is, the ESROM-based solu-

tion, involves the construction of a piece-wise surrogate model for the states of the nonlinear systems, based on the SROM of the input. The accuracy and the efficiency of the two methods are discussed in terms of the number of dynamic analyses required for obtaining the reliable response statistics. Results show that the SROM method is very efficient producing reliable results within an acceptable error with respect to $\mathrm{MC}$, with a small number of dynamic analyses, but it fails to represent statistics at the extremes. The ESROM is superior to the SROM method, and it outperforms in terms of computational time the MC method for some statistics, such as extreme-value distributions, or joint distributions of the input-response maxima.

Acknowledgements The work presented in this paper has been supported by the Marie Skłodowska-Curie Actions of the Euro- 
pean Union's Horizon 2020 Program under the grant agreement 704679-PARTNER. This support is gratefully acknowledged.

Funding This study was funded by the Marie Skłodowska-Curie Actions of the European Union's Horizon 2020 Program under the Grant Agreement No. 704679-PARTNER.

\section{Compliance with ethical standards}

Conflict of interest The author declares that he has no conflict of interest.

Open Access This article is distributed under the terms of the Creative Commons Attribution 4.0 International License (http:// creativecommons.org/licenses/by/4.0/), which permits unrestricted use, distribution, and reproduction in any medium, provided you give appropriate credit to the original author(s) and the source, provide a link to the Creative Commons license, and indicate if changes were made.

\section{References}

1. Soong, T., Grigoriu, M.: Random Vibration of Mechanical and Structural Systems. PTR Prentice-Hall Inc, Upper Saddle River (1993)

2. Field Jr., R., Grigoriu, M.: Optimal stochastic models for spacecraft atmospheric re-entry. J. Sound Vib. 290(3), 9911014 (2006)

3. Chen, X., Kareem, A.: Nonlinear response analysis of longspan bridges under turbulent winds. J. Wind Eng. Ind. Aerodyn. 89(14), 1335-1350 (2001). (Bluff Body Aerodynamics and Applications)

4. Radu, A., Grigoriu, M.: An earthquake-source-based metric for seismic fragility analysis. Bull. Earthq. Eng. 16, 37713789 (2018)

5. Yin, Y., Neild, S.A., Jiang, J.Z., Knowels, J.A.C., Nie, H.: Optimization of a main landing gear locking mechanism using bifurcation analysis. J. Aircr. 54(6), 2126-2139 (2017)

6. Grigoriu, M.: Stochastic Calculus: Applications in Science and Engineering. Birkhäuser, Boston (2002)

7. Shadab Far, M., Wang, Y.: Approximation of the Monte Carlo sampling method for reliability analysis of structures. Math. Probl. Eng. 2016, 1-9 (2016)

8. Majda, A.J., Qi, D.: Strategies for reduced-order models for predicting the statistical responses and uncertainty quantification in complex turbulent dynamical systems. SIAM Rev. 60(3), 491-549 (2018)

9. Caflisch, R.E.: Monte Carlo and quasi-Monte Carlo methods. Acta Numer. 7, 1-49 (1998)

10. Kocis, L., Whiten, W.J.: Computational investigations of low-discrepancy sequences. ACM Trans. Math. Softw. 23, 266-294 (1997)

11. Yang, X., Choi, M., Lin, G., Karniadakis, G.E.: Adaptive ANOVA decomposition of stochastic incompressible and compressible flows. J. Comput. Phys. 231, 1587-1614 (2012)

12. Stein, M.: Large sample properties of simulations using latin hypercube sampling. Technometrics 29(2), 143-151 (1987)
13. Li, X., Du, D., Pei, J., Menhas, M.: Probabilistic load flow calculation with latin hypercube sampling applied to gridconnected induction wind power system. Trans. Inst. Meas. Control 35(1), 56-65 (2013)

14. Roberts, J.B., Spanos, P.D.: Random Vibration and Statistical Linearization. Courier Corporation, Chelmsford (2003)

15. Elishakoff, I.: Method of Stochastic Linearization Revised and Improved, pp. 101-111. Springer, Dordrecht (1991)

16. Zhang, R., Elishakoff, I., Shinozuka, M.: Analysis of nonlinear sliding structures by modified stochastic linearization methods. Nonlinear Dyn. 5, 299-312 (1994)

17. Roberts, J., Spanos, P.: Stochastic averaging: an approximate method of solving random vibration problems. Int. J. NonLinear Mech. 21(2), 111-134 (1986)

18. Tien, W.-M., Namachchivaya, N.S., Coppola, V.T.: Stochastic averaging using elliptic functions to study nonlinear stochastic systems. Nonlinear Dyn. 4, 373-387 (1993)

19. Hijawi, M., Ibrahim, R.A., Moshchuk, N.: Nonlinear random response of ocean structures using first- and secondorder stochastic averaging. Nonlinear Dyn. 12, 155-197 (1997)

20. He, J.-H.: Homotopy perturbation method: a new nonlinear analytical technique. Appl. Math. Comput. 135(1), 73-79 (2003)

21. Spencer Jr., B.F., Bergman, L.A.: On the numerical solution of the Fokker-equations for nonlinear stochastic systems. Nonlinear Dyn. 4, 357-372 (1993)

22. Muscolino, G., Riccardi, G., Vasta, M.: Stationary and nonstationary probability density function for non-linear oscillator. Int. J. Non-Linear Mech. 32(6), 1051-1064 (1997)

23. Narayanana, S., Kumar, P.: Numerical solutions of FokkerPlanck equation of nonlinear systems subjected to random and harmonic excitations. Probab. Eng. Mech. 27(1), 35-46 (2012)

24. Wojtkiewicz, S.F., Spencer Jr., B.F., Bergman, L.A.: On the cumulant-neglect closure method in stochastic dynamics. Int. J. Non-Linear Mech. 31(5), 657-684 (1996)

25. Wojtkiewicz, S.F., Bergman, L.A.: A moment specification algorithm for control of nonlinear systems driven by Gaussian white noise. Int. J. Non-Linear Mech. 24(1), 17-30 (2001)

26. Li, R., Ghanem, R.: Adaptive polynomial chaos expansions applied to statistics of extremes in nonlinear random vibration. Probab. Eng. Mech. 13, 125-136 (1998)

27. Xiu, D., Lucor, D., Su, C.H., Karniadakis, G.E.: Stochastic modeling of flow-structure interactions using generalized polynomial chaos. J. Fluids Eng. 124(1), 51-59 (2002)

28. Sapsis, T.P., Majda, A.J.: Statistically accurate low-order models for uncertainty quantification in turbulent dynamical systems. Proc. Natl. Acad. Sci. USA 110(34), 13705-13710 (2013)

29. Kuether, R.J., Deaner, B.J., Hollkamp, J.J., Allen, M.S.: Evaluation of geometrically nonlinear reduced-order models with nonlinear normal modes. AIAA J. 53(11), 3273-3285 (2015)

30. Haller, G., Ponsioen, S.: Nonlinear normal modes and spectral submanifolds: existence, uniqueness and use in model reduction. Nonlinear Dyn. 86, 1-42 (2016)

31. Majda, A.J., Branicki, M.: Lessons in uncertainty quantification for turbulent dynamical systems. Discrete Contin. Dyn. Syst. 32, 3133-3221 (2012) 
32. Hill, T.L., Neild, S.A., Cammarano, A.: An analytical approach for detecting isolated periodic solution branches in weakly nonlinear structures. J. Sound Vib. 379, 150-165 (2016)

33. Pradlwarter, H.J., Schueller, G.I.: On advanced Monte Carlo simulation procedure in stochastic structural dynamics. Int. J. Non-Linear Mech. 32(4), 735-744 (1997)

34. Majda, A., Wang, X.: Nonlinear Dynamics and Statistical Theories for Basic Geophysical Flows. Cambridge University Press, Cambridge (2006)

35. Grigoriu, M.: Reduced order models for random functions. Application to stochastic problems. Appl. Math. Model. 33(1), 161-175 (2009)

36. Grigoriu, M.: Linear random vibration by stochastic reduced-order models. Int. J. Numer. Methods Eng. 82(12), 1537-1559 (2010)

37. Grigoriu, M.: A method for solving stochastic equations by reduced order models and local approximations. J. Comput. Phys. 231(19), 6496-6513 (2012)

38. Uy, W.I.T., Grigoriu, M.D.: An adaptive method for solving stochastic equations based on interpolants over voronoi cells. Probab. Eng. Mech. 51, 23-41 (2018)

39. Fei, Z., Huang, Y., Zhou, J., Xu, Q.: Uncertainty quantification of crosstalk using stochastic reduced order models. IEEE Trans. Electromagn. Compat. 59, 228-239 (2017)

40. Sarkar, S., Warner, J.E., Aquino, W., Grigoriu, M.D.: Stochastic reduced order models for uncertainty quantification of intergranular corrosion rates. Corros. Sci. 80, 257268 (2014)

41. Warner, J.E., Aquino, W., Grigoriu, M.D.: Stochastic reduced order models for inverse problems under uncertainty. Comput. Methods Appl. Mech. Eng. 285, 488-514 (2015)

42. Institutes, B.S.: Exctracts from Eurocode 8: design of structures for earthquake resistance. In: Structures Eurocodes PP 1990:2010_Extracts from the Structural Eurocodes for students of structural design (2010)

43. Agency, E.A.S.: Book2: Section AMC-Subpart F. In: Certification Specifications and Acceptable Means of Compliance for Large Aeroplanes CS-25 (2017)

44. Radu, A., Grigoriu, M.: A site-specific ground-motion simulation model: application for Vrancea earthquakes. Soil Dyn. Earthq. Eng. 111, 77-86 (2018)

45. Muller, P., Garrett, C., Osborne, A.: Rogue waves. Oceanography 18(3), 66-75 (2005)

46. Spence, S., Gioffre, M.: Large scale reliability-based design optimization of wind excited tall buildings. Probab. Eng. Mech. 28, 206-215 (2012)

47. Stathopoulos, T.: PDF of wind pressures on low-rise buildings. J. Struct. Div. ASCE 106, 973-990 (1980)

48. Ochi, M.K., Malakar, S.B., Wang, W.-C.: Statistical analysis of coastal waves observed during the ARSLOE project. UFL/COEL/TR-045, Coastal and Oceanographic Engineering Department, University of Florida, Gainesville, FL (1982)
49. Grigoriu, M.: Applied Non-Gaussian Processes: Examples, Theory, Simulation, Linear Random Vibration, and MATLAB Solutions, Chap. 2, pp. 5-40. Prentice-Hall, Englewood Cliffs (1995)

50. Grigoriu, M.: Simulation of stationary non-gaussian translation processes. J. Eng. Mech. 124(2), 121-126 (1998)

51. Lenci, S., Rega, G.: Optimal control of nonregular dynamics in a duffing oscillator. Nonlinear Dyn. 33, 71-86 (2003)

52. Leng, X.L., Wu, C.L., Ma, X.P., Meng, G., Fang, T.: Bifurcation and chaos analysis of stochastic duffing system under harmonic excitations. Nonlinear Dyn. 42, 185-198 (2005)

53. Shaw, A.D., Neild, S.A., Wagg, D.J.: Dynamic analysis of high static low dynamic stiffness vibration isolation mounts. J. Sound Vib. 332(6), 1437-1455 (2013)

54. Ikhouane, F., Rodellar, J.: On the hysteretic Bouc-Wen model. Nonlinear Dyn. 42, 79-95 (2005)

55. Ikhouane, F., Hurtado, J.E., Rodellar, J.: Variation of the hysteresis loop with the Bouc-Wen model parameters. Nonlinear Dyn. 48, 361-380 (2007)

56. Goda, K., Hong, H.P., Lee, C.S.: Probabilistic characteristics of seismic ductility demand of SDOF systems with Bouc-Wen hysteretic behavior. J. Earthq. Eng. 13(5), 600622 (2009)

57. Vakakis, A.F.: Inducing passive nonlinear energy sinks in vibrating systems. J. Vib. Acoust. 123(3), 324-332 (2001)

58. Joo, H.K., Mohamad, M.A., Sapsis, T.P.: Extreme events and their optimal mitigation in nonlinear structural systems excited by stochastic loads: application to ocean engineering systems. Ocean Eng. 142, 145-160 (2017)

59. Warner, J.E., Grigoriu, M., Aquino, W.: Stochastic reduced order models for random vectors: application to random eigenvalue problems. Probab. Eng. Mech. 31, 1-11 (2013)

60. Allen, I., Miller, C., Rice, T.R.: Discrete approximations of probability distributions. Manag. Sci. 29(3), 352-362 (1983)

61. Walser, J.P.: Integer Optimization by Local Search. Springer, New York (1999)

62. Stoyan, D., Kendall, W.S., Mecke, J.: Stochastic Geometry and Its Applications. Wiley, New York (1987). Chap. 5.4

63. Duda, R.O., Hart, P.E., Stork, D.G.: Pattern Classification, 2nd edn. Wiley, New York (2001). Chap. 10

64. Huang, S.P., Quek, S.T., Phoon, K.K.: Convergence study of the truncated Karhunen-Loeve expansion for simulation of stochastic processes. Int. J. Numer. Methods Eng. 52(9), 1029-1043 (2001)

Publisher's Note Springer Nature remains neutral with regard to jurisdictional claims in published maps and institutional affiliations. 